\title{
Global Existence and Boundedness of Large Solutions to Nonlinear Equations of Viscoelasticity with Hardening
}

\author{
H. D. Alber
}

Fachbereich Mathematik der Technischen Hochschule Darmstadt, Schloßgartenstraße 7, D-64289 Darmstadt, Germany

Received: 6 September 1993

Dedicated to Erhard Meister on the occasion of his sixtyfifth birthday

Abstract: For the solutions of an initial-boundary value problem for the equations of viscoelasticity with isotropic hardening we derive a uniform bound under a growth condition for the nonlinearities in the case of one-space dimension. Global-in-time existence of solutions to large initial data is a consequence of the existence of this bound. In the most simple form, the equations we consider are

$$
\begin{aligned}
\left(\partial_{t}+\partial_{x}\right) \alpha & =\frac{1}{2} g_{1}\left(|\beta-\alpha-s|, z_{1}\right)(\beta-\alpha-s), \\
\left(\partial_{t}-\partial_{x}\right) \beta & =-\frac{1}{2} g_{1}\left(|\beta-\alpha-s|, z_{1}\right)(\beta-\alpha-s), \\
\partial_{t} s & =g_{1}\left(|\beta-\alpha-s|, z_{1}\right)(\beta-\alpha-s)-g_{2}\left(|s|, z_{2}\right) s, \\
\partial_{t} z & =\partial_{t}\left(z_{1}, z_{2}\right)=h(z,|\beta-\alpha-s|,|s|),
\end{aligned}
$$

with suitable functions $g_{1}, g_{2}, h$ satisfying $g_{2} \geq 0$ and

$$
0 \leq g_{1}(\eta, \zeta) \leq M_{1} \eta^{\varrho}+M_{2}, \quad \varrho<2 .
$$

\section{Introduction}

To study the viscoelastic behavior of metals frequently constitutive models are used whose derivation is based on the hypothesis that a set of internal variables exist which together with the stress- and strain tensors completely characterize the state of the material. In this paper we investigate an initial-boundary value problem for a system of partial and ordinary differential equations resulting from this hypothesis and from the hypothesis of small deformations. The constitutive model leading to these differential equations is shortly presented in the appendix of this paper.

It is known that when the right-hand side of the system of ordinary differential equations (A3)-(A5) stated in the appendix is the gradient of a convex functional,

This research was partially supported by the DFG-Forschergruppe "Mathematische und Ingenieurwissenschaftliche Analyse bruchmechanischer und inelastischer Probleme" 
then the theory of monotone operators can be used to prove existence of solutions for the initial-boundary value problem. This gradient condition is automatically satisfied in the case when isotropic hardening is not taken into account in the constitutive model. Accordingly, the monotone operator approach is used in $[2,3,4,6,8,12,13]$ to prove existence for models without parameters of isotropic hardening, and in [7] for models with isotropic hardening satisfying the gradient condition. More precisely, in [7] the elastic-perfectly plastic case is treated, but since this is a limit case of viscoelasticity, the proof generalizes immediately to viscoelastic models.

However, this gradient condition is not a consequence of the thermodynamic laws. In thermodynamics it is often assumed that a convex dissipation potential exists associated with the constitutive model, cf. [9, 11, 13], and under some additional assumptions the gradient condition follows from the existence of such a dissipation potential, for instance if the model contains only one parameter of isotropic hardening and satisfies some other conditions. In general however, neither does the gradient condition follow from the existence of a convex dissipation potential, nor is the existence of a dissipation potential itself a consequence of the thermodynamic laws, and actually, for most constitutive models from engineering the gradient condition is not satisfied, cf. for example [5, 10].

But since all models are dissipative, it seems probable that a global solution exists for the initial-boundary value problem also when the gradient condition is not satisfied and the monotone operator approach cannot be used. New arguments must be used in the proof of global existence, and in this paper we study this problem and consider the case when the deformations only depend on one space variable. Under some growth conditions for functions appearing in the set of constitutive equations $L^{\infty}$-bounds for the solutions of the resulting initial-boundary value problem to large initial data are derived. These bounds imply that the solutions exist globally in time.

Our investigations are motivated by viscoelasticity, but we believe that these $L^{\infty}$ bounds are of independent mathematical interest.

We now state the partial differential equations treated in this paper. To make these equations better understandable, we start with the equations in the full threedimensional form and subsequently reduce them to the one-dimensional form considered in this paper.

Let $\mathscr{S}^{3}$ be the space of symmetric $3 \times 3$ matrices, and let $v(x, t) \in \mathbb{R}^{3}$ be the velocity, $\sigma(x, t) \in \mathscr{S}^{3}$ the Cauchy stress tensor and $\varepsilon(x, t)=\frac{1}{2}[\nabla v(x, t)+$ $\left.(\nabla v(x, t))^{T}\right] \in \mathscr{S}^{3}$ the time derivative of the strain tensor at the point $x \in \mathbb{R}^{3}$ at time $t$. For tensors $e=\left(e_{i j}\right)_{i, j=1, \ldots, 3}, \hat{e}=\left(\hat{e}_{i j}\right)_{\imath, \jmath=1, \ldots, 3}$ we write

$$
(e, \hat{e})=\sum_{i, j=1}^{3} e_{\imath j} \hat{e}_{\imath j}, \quad|e|=(e, e)^{1 / 2} .
$$

Then in three dimensions the partial differential equations are

$$
\begin{aligned}
\hat{\varrho} \partial_{t} v & =\operatorname{div} \sigma \\
\partial_{t} \sigma & =D \varepsilon-g_{1}\left(|P \sigma-s|, z_{1}\right) D(P \sigma-s), \\
\partial_{t} s & =\mathscr{M} b g_{1}\left(|P \sigma-s|, z_{1}\right)(P \sigma-s)-\mathscr{M} g_{2}\left(|s|, z_{2}\right) s, \\
\partial_{t} z & =h(z,|P \sigma-s|,|s|) .
\end{aligned}
$$

Here $\varrho$ $>0$ is the density, which we assume to be constant, and $D: \mathscr{S}^{3} \rightarrow \mathscr{S}^{3}$ is the elasticity tensor, which is assumed to be constant, symmetric and positive definite. 
The operator $P: \mathscr{S}^{3} \rightarrow \mathscr{S}^{3}$ is defined by

$$
[P \sigma](x, t)=\sigma(x, t)-\frac{1}{3}(\operatorname{tr} \sigma(x, t)) I,
$$

where $I$ is the identity matrix. Thus $P \sigma$ is the stress deviator, and $P$ is the orthogonal projector from the space of symmetric tensors to the space of symmetric tensors with vanishing trace. The variable $s(x, t) \in \mathscr{S}^{3}$ is a parameter of kinematic hardening and has the dimensions of a stress, $z(x, t)=\left(z_{1}(x, t), z_{2}(x, t)\right) \in \mathbb{R}^{2}$ are parameters of isotropic hardening, $g_{1}, g_{2}: \mathbb{R}_{0}^{+} \times \mathbb{R} \rightarrow \mathbb{R}_{0}^{+}, h: \mathbb{R}^{2} \times\left(\mathbb{R}_{0}^{+}\right)^{2} \rightarrow \mathbb{R}^{2}$ are given functions which together with the constant $\mathscr{C} b>0$ and the elasticity tensor characterize the properties of the inelastic material.

We assume now that all functions in (1.1)-(1.4) only depend on the first component of $x$ and on $t$, and appropriately let $x$ denote now a real variable from the interval $[0, L]$ with a constant $L>0$. We also assume that $\hat{\varrho} \equiv 1$. Equations (1.1)-(1.4) can then be written in the form

$$
\begin{aligned}
\left(\begin{array}{c}
v \\
\sigma
\end{array}\right)_{t} & =\left(\begin{array}{cc}
0 & Q \\
D Q^{*} & 0
\end{array}\right)\left(\begin{array}{c}
v \\
\sigma
\end{array}\right)_{x}-\left(\begin{array}{c}
0 \\
g_{1}\left(|P \sigma-s|, z_{1}\right) D(P \sigma-s)
\end{array}\right), \\
\partial_{t} s & =\mathscr{O} g_{1}\left(|P \sigma-s|, z_{1}\right)(P \sigma-s)-\mathscr{C b} g_{2}\left(|s|, z_{2}\right) s, \\
\partial_{t} z & =h(z,|P \sigma-s|,|s|)
\end{aligned}
$$

where the linear operator $Q: \mathscr{S}^{3} \rightarrow \mathbb{R}^{3}$ is defined by

$$
Q \sigma=\left(\sigma_{\imath 1}\right)_{\imath=1,2,3}
$$

and $Q^{*}: \mathbb{R}^{3} \rightarrow \mathscr{S}^{3}$ is the adjoint of this operator. If $v \in \mathbb{R}^{3}$ and if $(v, 0,0)$ is the $3 \times 3$-matrix with columns $v, 0,0$, then

$$
Q^{*} v=\frac{1}{2}\left[(v, 0,0)+(v, 0,0)^{T}\right] .
$$

The remaining notations are as above. We also require

$$
\begin{gathered}
Q \sigma(0, t)=Q \sigma(L, t)=0, \quad t \geq 0, \\
v(x, 0)=v^{0}(x), \quad \sigma(x, 0)=\sigma^{0}(x), \\
s(x, 0)=s^{0}(x), \quad z(x, 0)=z^{0}(x) ; \quad 0 \leq x \leq L .
\end{gathered}
$$

Equations (1.6)-(1.11) define the initial-boundary value problem studied in this paper. The properties of the material modelled by (1.6)-(1.11) are specified by the choice of the functions $g_{1}, g_{2}$ and $h$, and in the engineering literature a wide variety of choices for these functions can be found, cf. $[5,10]$ for examples. In particular this is true for $g_{2}$ and $h$, whereas some specifications of $g_{1}$ are determined by basic properties of viscoelastic materials. A typical example is

$$
g_{1}\left(|P \sigma-s|, z_{1}\right)=C\left(|P \sigma-s| / z_{1}\right)^{m}
$$

with material parameters $C$ and $m \sim 5 \ldots 7$. In this example the function $h$ in (1.8) and the initial data $z^{0}$ must be given such that the solutions $z$ satisfy $z_{1}(x, t) \geq c(t)>0$. Often the functions $g_{1}, g_{2}, h$ are not even differentiable.

It is therefore desirable to prove existence of solutions of (1.6)-(1.11) for a class of functions $g_{1}, g_{2}, h$ as large as possible. Since $g_{1}, g_{2} \geq 0$, there is damping in (1.6)-(1.11), as follows from the energy estimate proved in Sect. 3. This suggests to use energy estimates to prove existence of solutions, and in [1] this has been done for a special choice of $g_{1}, g_{2}, h$. However, this approach seems to be severely restricted: First of all, since the equations are nonlinear, it required proofs of a-priori 
estimates for derivatives of the solution. But as is seen from [1], the proof of such estimates is difficult already for first derivatives in the case of one space dimension, and it strongly depends on the special form of $g_{1}, g_{2}, h$ chosen. Generally, because of physical and mathematical reasons it is not probable that solutions of (1.6)-(1.11) have many derivatives. And secondly, a fundamental difficulty is that the method of energy estimates only works for small initial data. Because of all these reasons, it seems desirable and even necessary to prove existence of solutions without recourse to estimate for derivatives whatsoever, and in this paper we give such a proof for the global in time existence of weak solutions of (1.6)-(1.11) to large initial data under growth conditions for $g_{1}$ and $h$. To state the main results of this paper we need two definitions:

Let $T>0$ or $T=\infty$. A function

$$
(v, \sigma, s, z):[0, L] \times[0, T) \rightarrow \mathbb{R}^{3} \times \mathscr{S}^{3} \times \mathscr{S}^{3} \times \mathbb{R}^{2}
$$

is called a continuous weak solution of (1.6)-(1.11) in $[0, L] \times[0, T)$ if $(v, \sigma, s, z) \in$ $C([0, L] \times[0, T))$ and if $(v, \sigma, s, z)$ is a weak solution of (1.6)-(1.8) satisfying (1.10) and (1.11).

Let $\Omega$ be a nonempty subset of $\mathbb{R}^{n}$. We call a function $f: \Omega \rightarrow \mathbb{R}$ locally Lipschitz continuous if to every compact subset $K$ of $\Omega$ there exists a constant $C_{K}$ with

$$
|f(x)-f(y)| \leq C_{K}|x-y|
$$

for all $x, y \in K$.

The main result is

Theorem 1.1. Let the functions $g_{1}, g_{2}: \mathbb{R}_{0}^{+} \times \mathbb{R} \rightarrow \mathbb{R}_{0}^{+}, h: \mathbb{R}^{2} \times\left(\mathbb{R}_{0}^{+}\right)^{2} \rightarrow \mathbb{R}^{2}$ be locally Lipschitz continuous, and assume that there are constants $M_{1}^{*}, M_{2}^{*}, \varrho>0$ with

$$
g_{1}(\eta, \zeta) \leq M_{1}^{*} \eta^{\varrho}+M_{2}^{*}
$$

for all $\eta \geq 0, \zeta \in \mathbb{R}$, where

$$
0<\varrho<1
$$

or

$$
0<\varrho<2 \text { and } g_{2} \equiv 0 \text {. }
$$

Then there exist constants $M_{3}^{*}, M_{4}^{*}, k_{0}, \Lambda>0$ such that to all initial data

$$
\left(v^{0}, \sigma^{0}, s^{0}, z^{0}\right) \in C\left([0, L], \mathbb{R}^{3} \times \mathscr{S}^{3} \times \mathscr{S}^{3} \times \mathbb{R}^{2}\right)
$$

satisfying

$$
Q \sigma^{0}(0)=Q \sigma^{0}(L)=0 ; \quad \operatorname{tr} s^{0}(x)=0 \text { for all } 0 \leq x \leq L,
$$

there is $T>0$ and a continuous weak solution $(v, \sigma, s, z)$ of $(1.6)-(1.11)$ in $[0, L] \times$ $[0, T)$. This solution satisfies for all $0 \leq t<T$,

$$
\begin{aligned}
\sup _{0 \leq x \leq L}|(v, \sigma, s)(x, t)| \leq \max \{ & M_{3}^{*}\left[1+K^{*}(t, E(0))\right]\left|\left(v^{0}, \sigma^{0}, s^{0}\right)\right|_{\infty}, \\
& {\left.\left[M_{4}^{*} K^{*}(t, E(0))\left|\left(v^{0}, \sigma^{0}, s^{0}\right)\right|_{\infty}\right]^{\gamma}\right\} }
\end{aligned}
$$

where

$$
\begin{aligned}
\left|\left(v^{0}, \sigma^{0}, s^{0}\right)\right|_{\infty} & =\sup _{0 \leq x \leq L}\left(\left|v^{0}(x)\right|^{2}+\left|\sigma^{0}(x)\right|^{2}+\left|s^{0}(x)\right|^{2}\right)^{1 / 2} \\
E(0) & =\frac{1}{2} \int_{0}^{L}\left|v^{0}(x)\right|^{2}+\left(D^{-1} \sigma^{0}(x), \sigma^{0}(x)\right)+\frac{1}{\mathscr{M b}}\left|s^{0}(x)\right|^{2} d x
\end{aligned}
$$


and

$$
K^{*}(t, E(0))=\hat{K}^{\frac{\varrho}{2+\varrho}} \sum_{m=0}^{\infty} \frac{2\left(k_{0}\right)^{m+1}[\Lambda(E(0)+L)]^{\frac{\varrho}{2+\varrho}}(t+1)^{m+\frac{2}{2+\varrho}}}{\prod_{\nu=1}^{m}\left[1+\left(\nu-\frac{\varrho}{2+\varrho}\right) \frac{2+\varrho}{2-\varrho}\right]^{\frac{2-\varrho}{2+\varrho}}}
$$

For the constants $\gamma$ in (1.15) and $\hat{K}$ in (1.16) we have in case that (1.13a) holds

$$
\gamma=\frac{1}{1-\varrho}, \quad \hat{K}=t
$$

and in case that $(1.13 \mathrm{~b})$ holds

$$
\gamma=\frac{2+\varrho}{2-\varrho}, \quad \hat{K}=\frac{\varrho}{(2+\varrho) \mu_{0}}
$$

with a suitable constant $\mu_{0}>0$.

The solution $(v, \sigma, s, z)$ is locally unique, that is if $\left(v^{(\imath)}, \sigma^{(i)}, s^{(i)}, z^{(i)}\right)$ are continuous weak solutions of (1.6)-(1.11) on $[0, L] \times\left[0, T_{2}\right)$ to the same initial data for $i=1,2$, then $\left(v^{(1)}, \sigma^{(1)}, s^{(1)}, z^{(1)}\right)=\left(v^{(2)}, \sigma^{(2)}, s^{(2)}, z^{(2)}\right)$ on $[0, L] \times\left[0, \min \left(T_{1}, T_{2}\right)\right)$.

Note that the series in (1.16) converges, since for all sufficiently large $m$

$$
\left\{\prod_{\nu=1}^{m}\left[1+\left(\nu-\frac{\varrho}{2+\varrho}\right) \frac{2+\varrho}{2-\varrho}\right]\right\}^{\frac{2-\varrho}{2+\varrho}} \geq\left\{\prod_{\nu=1}^{m} \nu\right\}^{\frac{2-\varrho}{2+\varrho}}=(m !)^{\frac{2-\varrho}{2+\varrho}} \geq\left(\frac{m}{e}\right)^{\frac{2-\varrho}{2+\varrho} m}
$$

by Stirling's formula.

The components $(v, \sigma, s)$ of a local solution of (1.6)-(1.11) are thus contained in the space $L^{\infty}([0, L] \times[0, T))$ for every $0<T<\infty$. It is a standard result that local solutions can be continued as long as they stay bounded in $L^{\infty}$, which means that solutions of (1.6)-(1.11) can be continued as long as the hardening parameter $z$ remains bounded. In the engineering models normally the function $h$ in Eq. (1.8) is of such a form that $z$ remains bounded, but from the only assumption we made for $h$, namely that $h$ is locally Lipschitz continuous, one cannot conclude this. The following simple assumption, normally satisfied in models of viscoelasticity, is sufficient to guarantee boundedness of $z$ :

Corollary 1.2. Let the hypotheses of Theorem 1.1 be satisfied and assume that there exists a monotonically increasing function $c^{*}: \mathbb{R}_{0}^{+} \rightarrow \mathbb{R}_{0}^{+}$with

$$
\left|h\left(\zeta, \eta_{1}, \eta_{2}\right)\right| \leq c^{*}\left(\eta_{1}+\eta_{2}\right)(|\zeta|+1)
$$

for all $\left(\zeta, \eta_{1}, \eta_{2}\right) \in \mathbb{R}^{2} \times\left(\mathbb{R}_{0}^{+}\right)^{2}$. Then to all initial data

$$
\left(v^{0}, \sigma^{0}, s^{0}, z^{0}\right) \in C\left([0, L], \mathbb{R}^{3} \times \mathscr{S}^{3} \times \mathscr{S}^{3} \times \mathbb{R}^{2}\right)
$$

satisfying (1.14) there exists a locally unique, continuous weak solution of (1.6)-(1.11) in $[0, L] \times[0, \infty)$. The solution is contained in $L^{\infty}([0, L] \times[0, T))$ for every $0<T<\infty$ and satisfies (1.15).

The estimate (1.15) is the main result of this paper, and as already noted, we believe that this result is of mathematical interest beyond the application to the equations of viscoelasticity. Therefore we state it here in the most simple form: Consider the totally one-dimensional case with $v, \sigma, s \in \mathbb{R}$ and $D=1$, set $\mathscr{C} b=1$, drop the operator $P$, 
introduce the Riemann invariants $\alpha=\frac{1}{2}(v-\sigma), \beta=\frac{1}{2}(v+\sigma)$, and assume $z(x, t)$ to be a known function. Then (1.6), (1.7) reduce to

$$
\begin{aligned}
\left(\partial_{t}+\partial_{x}\right) \alpha & =\frac{1}{2} g_{1}(|\beta-\alpha-s|, x, t)(\beta-\alpha-s), \\
\left(\partial_{t}-\partial_{x}\right) \beta & =-\frac{1}{2} g_{1}(|\beta-\alpha-s|, x, t)(\beta-\alpha-s), \\
\partial_{t} s & =g_{1}(|\beta-\alpha-s|, x, t)(\beta-\alpha-s)-g_{2}(|s|, x, t) s .
\end{aligned}
$$

Basically, what we prove is that solutions of this system satisfy the estimate (1.15) if (1.12) is satisfied with $\varrho<2$. In this simpler case it is not necessary to require $g_{2} \equiv 0$ when $1 \leq \varrho<2$.

From the example given above it is seen that for many engineering models the condition $\varrho<1$ or $\varrho<2$ imposed by (1.13a), (1.13b) is too restrictive. We do not know whether this condition is necessary for the boundedness of solutions, nor do we know whether global solutions can be found in a function space larger than $L^{\infty}$ if this condition is not satisfied.

The paper is organized as follows: In Sect. 2 we transform the initial-boundary value problem into an equivalent periodic Cauchy problem in characteristic form. In Theorem 2.5 and Corollary 2.6, Theorem 1.1 and Corollary 1.2 are restated for the reformulated version of the problem. The remaining sections are devoted to the proofs of Theorem 2.5 and Corollary 2.6. In Sect. 3 we state the local existence theorem and prove an energy inequality, which is needed in the proof of the a-priori estimate (1.15). In Sect. 4 and 5 the proof of this a-priori estimate in the reformulated version is given. Section 4 contains some preparatory results and Sect. 5 the final proof of the a-priori estimate, and at the end, the proofs of Theorem 2.5 and Corollary 2.6.

\section{Reformulation of the Problem}

In this section we reformulate the problem in the form of an equivalent characteristic and periodic Cauchy problem, and at the end of the section we state Theorem 2.5 and Corollary 2.6, which are the equivalent versions of Theorem 1.1 and Corollary 1.2 for the reformulated problem.

In a first step we transform the problem (1.6)-(1.11) into a periodic Cauchy problem. To this end we define some function spaces. For $u=(v, \sigma, s, z)$, $\hat{u}=(\hat{v}, \hat{\sigma}, \hat{s}, \hat{z}) \in \mathbb{R}^{3} \times \mathscr{S}^{3} \times \mathscr{S}^{3} \times \mathbb{R}^{2}$ we define a scalar product

$$
[u, \hat{u}]=(v, \hat{v})+\left(D^{-1} \sigma, \hat{\sigma}\right)+\frac{1}{\mathscr{M b}^{6}}(s, \hat{s})+(z, \hat{z}),
$$

where $(w, \hat{w})=\sum_{i=1}^{n} w_{i} \hat{w}_{i}$ and $(\sigma, \hat{\sigma})=\sum_{i, \jmath=1}^{3} \sigma_{i j} \hat{\sigma}_{\imath \jmath}$ when $w, \hat{w} \in \mathbb{R}^{n}, \sigma, \hat{\sigma} \in \mathscr{S}^{3}$.

To define scalar products and norms on the spaces $\mathbb{R}^{3} \times \mathscr{S}^{3}, \mathbb{R}^{3} \times \mathscr{S}^{3} \times \mathscr{S}^{3}, \mathscr{S}^{3} \times \mathbb{R}^{2}$ we identify these spaces with the subspaces $\mathbb{R}^{3} \times \mathscr{S}^{3} \times\{0\} \times\{0\}, \mathbb{R}^{3} \times \mathscr{S}^{3} \times \mathscr{S}^{3} \times\{0\}$, $\{0\} \times\{0\} \times \mathscr{S}^{3} \times \mathbb{R}^{2}$ of $\mathbb{R}^{3} \times \mathscr{S}^{3} \times \mathscr{S}^{3} \times \mathbb{R}^{2}$ and use the scalar product induced by $[u, \hat{u}]$ on these subspaces. We also denote these scalar products by $[\cdot, \cdot \cdot]$. The norms belonging to these scalar products on the different spaces are denoted by

$$
\|u\|=[u, u]^{1 / 2} .
$$


Let $\mathscr{B}(\mathbb{R})$ be the space of all functions $(v, \sigma, s, z): \mathbb{R} \rightarrow \mathbb{R}^{3} \times \mathscr{S}^{3} \times \mathscr{S}^{3} \times \mathbb{R}^{2}$ with

$$
\begin{aligned}
& (v, \sigma, s, z) \in C([0, L]) \\
& (v, \sigma, s, z)(x)=(v,-\sigma-s, z)(-x), \quad x \neq n L \text { for all integers } n, \\
& (v, \sigma, s, z)(x+2 L)=(v, \sigma, s, z)(x) \\
& Q \sigma(0)=Q \sigma(L)=0 .
\end{aligned}
$$

These conditions imply that $v, Q \sigma$ and $z$ are continuous on $\mathbb{R}$. For $T>0$ or $T=\infty$ let $\mathscr{B}(\mathbb{R} \times[0, T))$ denote the space of all functions $(v, \sigma, s, z): \mathbb{R} \times[0, T) \rightarrow$ $\mathbb{R}^{3} \times \mathscr{S}^{3} \times \mathscr{S}^{3} \times \mathbb{R}^{2}$ with

$$
(v, \sigma, s, z) \in C([0, L] \times[0, T))
$$

such that

$$
(v, \sigma, s, z)(\cdot, t) \in \mathscr{B}(\mathbb{R})
$$

for every $t \in[0, T)$.

Finally, let $A: \mathbb{R}^{3} \times \mathscr{S}^{3} \rightarrow \mathbb{R}^{3} \times \mathscr{S}^{3}$ denote the operator

$$
A=\left(\begin{array}{cc}
0 & -Q \\
-D Q^{*} & 0
\end{array}\right)
$$

with $Q$ defined in (1.9).

Lemma 2.1. Let $(v, \sigma, s, z) \in C\left([0, L] \times[0, T), \mathbb{R}^{3} \times \mathscr{S}^{3} \times \mathscr{S}^{3} \times \mathbb{R}^{2}\right)$ be a continuous weak solution of (1.6)-1.11) to the initial data $\left(v^{0}, \sigma^{0}, s^{0}, z^{0}\right)$ satisfying (1.14), and let $(\tilde{v}, \tilde{\sigma}, \tilde{s}, \tilde{z})$ denote the unique extension of $(v, \sigma, s, z)$ to $\mathbb{R} \times[0, T)$ contained in $\mathscr{C}(\mathbb{R} \times[0, T))$. Let $\left(\tilde{v}^{0}, \tilde{\sigma}^{0}, \tilde{s}^{0}, \tilde{z}^{0}\right)(x)=(\tilde{v}, \tilde{\sigma}, \tilde{s}, \tilde{z})(x, 0)$, and $\tilde{w}=(\tilde{v}, \tilde{\sigma}), \tilde{w}^{0}=$ $\left(\tilde{v}^{0}, \tilde{\sigma}^{0}\right)$. Then $(\tilde{w}, \tilde{s}, \tilde{z})$ is a weak solution of

$$
\begin{aligned}
\tilde{w}_{t}+A \tilde{w}_{x} & =-\left(\begin{array}{c}
0 \\
g_{1}\left(|P \tilde{\sigma}-P \tilde{s}|, \tilde{z}_{1}\right) D(P \tilde{\sigma}-P \tilde{s})
\end{array}\right) \\
\partial_{t} \tilde{s} & =\mathscr{M} g_{1}\left(|P \tilde{\sigma}-P \tilde{s}|, \tilde{z}_{1}\right)(P \tilde{\sigma}-P \tilde{s})-\mathscr{M} g_{2}\left(|P \tilde{s}|, \tilde{z}_{2}\right) P \tilde{s}, \\
\partial_{t} \tilde{z} & =h(\tilde{z},|P \tilde{\sigma}-P \tilde{s}|,|P \tilde{s}|) \\
\tilde{w}(x, 0) & =\tilde{w}^{0}(x), \quad \tilde{s}(x, 0)=\tilde{s}^{0}(x), \quad \tilde{z}(x, 0)=\tilde{z}^{0}(x) .
\end{aligned}
$$

Conversely, if $(\tilde{w}, \tilde{s}, \tilde{z}) \in \mathscr{B}(\mathbb{R} \times[0, T))$ is a weak solution of (2.10)-(2.13) with initial data satisfying

$$
\operatorname{tr} \tilde{s}^{0}(x)=0
$$

for all $x \in \mathbb{R}$, then the restriction $(w, s, z)$ of $(\tilde{w}, \tilde{s}, \tilde{z})$ to $[0, L] \times[0, T)$ is a continuous weak solution of (1.6)-(1.11) with initial data

$$
\left(w^{0}, s^{0}, z^{0}\right)=\left(\tilde{w}^{0}, \tilde{s}^{0}, \tilde{z}^{0}\right)_{\mid[0, L]} .
$$

Proof. If $(v, \sigma, s, z)$ is a continuous weak solution of (1.6)-(1.11) with initial data satisfying (1.14), then (1.7) implies

$$
\partial_{t}(\operatorname{tr} s)=-\mathscr{M} g_{1}\left(|P \sigma-s|, z_{1}\right)(\operatorname{tr} s)-\mathscr{M} b g_{2}\left(|s|, z_{2}\right) \operatorname{tr} s
$$

in the weak sense in $(0, L) \times[0, T)$, since $\operatorname{tr} P \sigma=0$, and

$$
\operatorname{tr} s(x, 0)=\operatorname{tr} s^{0}(x)=0, \quad x \in[0, L]
$$

hence

$$
\operatorname{tr} s(x, t)=0
$$


and therefore $P s(x, t)=s(x, t)$ for all $(x, t) \in[0, L] \times[0, T)$. Equations (1.6)-(1.8) thus hold in the weak sense if $s$ is replaced by $P s$ on the right-hand sides of these equations. On the other hand, if (2.10)-(2.14) hold, then (2.11) yields $\partial_{t}(\operatorname{tr} \tilde{s}(x, t))=0$ for all $(x, t) \in \mathbb{R} \times[0, T)$, which together with $\operatorname{tr} \tilde{s}(x, 0)=\operatorname{tr} \tilde{s}^{0}(x)=0$ yields $P \tilde{s}(x, t)=\tilde{s}(x, t)$, and we can replace $P \tilde{s}$ in $(2.10)-(2.12)$ by $\tilde{s}$. The other parts of the proof are standard, and we leave them to the reader.

In the next step we write (2.10)-(2.14) in characteristic form. The initial data and solution will be contained in function spaces $\mathscr{Y}(\mathbb{R})$ and $\mathscr{Y}(\mathbb{R} \times[0, T))$, which we define now.

As preparation for this definition we need some information about the operator $A$ defined in (2.9). Note that $A$ maps the space $\mathbb{R}^{3} \times \mathscr{S}^{3}$ of dimension nine into itself and is symmetric with respect to the scalar product $[w, \hat{w}]=(v, \hat{v})+\left(D^{-1} \alpha, \hat{\alpha}\right)$, where $w=(v, \alpha), \hat{w}=(\hat{v}, \hat{\alpha})$. Therefore $A$ has a complete orthonormal system $\left\{w_{i}\right\}$ of nine eigenvectors. To determine the eigenvectors and eigenvalues, let $\lambda$ be an eigenvalue and $w=(u, \alpha)$ an eigenvector of $A$. Then

$$
-Q \alpha=\lambda u ; \quad-D Q^{*} u=\lambda \alpha
$$

hence, for $\lambda \neq 0$,

$$
Q D Q^{*} u=\lambda^{2} u, \quad D Q^{*} Q \alpha=\lambda^{2} \alpha .
$$

The operator $Q D Q^{*}$ is symmetric and positive definite on $\mathbb{R}^{3}$, hence has eigenvalues $0<\mu_{1} \leq \mu_{2} \leq \mu_{3}$ counted according to multiplicity, and a set of eigenvectors $r_{1}, r_{2}, r_{3}$ satisfying

$$
\left(r_{i}, r_{\jmath}\right)=\frac{1}{2} \delta_{\imath \jmath}
$$

Lemma 2.2. The eigenvalues $\lambda_{-4}, \ldots, \lambda_{4}$ of $A$, counted according to multiplicity, are

$$
\lambda_{i}= \begin{cases}-\sqrt{\mu_{-i-1}}, & i=-4,-3,-2 \\ 0, & i=-1,0,1 \\ \sqrt{\mu_{\imath-1}}, & i=2,3,4\end{cases}
$$

Let $\left\{w_{i}\right\}_{i=-4}^{4}$ be an orthonormal system of eigenvectors of $A$ with $w_{i}$ corresponding to $\lambda_{i}$. Then

$$
w_{\imath}= \begin{cases}\left(r_{|\imath|-1},-\frac{1}{\lambda_{\imath}} D Q^{*} r_{|i|-1}\right), & \text { if } 2 \leq|i| \leq 4 \\ \left(0, \alpha_{i}\right) & \text { if } i=-1,0,1,\end{cases}
$$

where $\alpha_{-1}, \alpha_{0}, \alpha_{1} \in \mathscr{S}^{3}$ form a basis of the space $\operatorname{ker}(Q)$ of dimension three and satisfy $\left(D^{-1} \alpha_{\imath}, \alpha_{\jmath}\right)=\delta_{\imath \jmath}$ for $-1 \leq i, j \leq 1$.

Proof. All statements follow immediately from (2.15)-(2.17).

In the set theoretic sense, that is, not counted according to multiplicity, let

$$
\lambda^{\left(-k_{0}\right)}<\lambda^{\left(-k_{0}+1\right)}<\ldots<\lambda^{\left(k_{0}\right)}
$$

be the eigenvalues of $A$. Then $k_{0} \leq 3, \lambda^{(-k)}=-\lambda^{(k)}$, and $\lambda^{(0)}=0$. Let $Y_{k} \subset \mathbb{R}^{3} \times \mathscr{S}^{3}$ be the eigenspace of $A$ to the eigenvalue $\lambda^{(k)}$, and let $\Pi_{k}$ be the projector from $\mathbb{R}^{3} \times \mathscr{S}^{3}$ onto $Y_{k}$ orthogonal with respect to the scalar product $[w, \hat{w}]$. Then the 
space $Y_{-k_{0}} \times \ldots \times Y_{k_{0}}$ is isomorphic to the space $\mathbb{R}^{3} \times \mathscr{S}^{3}$ and the mapping

$$
w \rightarrow\left(\Pi_{-k_{0}} w, \ldots, \Pi_{k_{0}} w\right): \mathbb{R}^{3} \times \mathscr{S}^{3} \rightarrow Y_{-k_{0}} \times \ldots \times Y_{k_{0}}
$$

is an isomorphism with inverse

$$
\left(w^{\left(-k_{0}\right)}, \ldots, w^{\left(k_{0}\right)}\right) \rightarrow w=\sum_{k=-k_{0}}^{k_{0}} w^{(k)}
$$

if we define the scalar product on the new space by

$$
\left[\left(w^{\left(-k_{0}\right)}, \ldots, w^{\left(k_{0}\right)}\right),\left(\hat{w}^{\left(-k_{0}\right)}, \ldots, \hat{w}^{\left(k_{0}\right)}\right)\right]=\sum_{k=-k_{0}}^{k_{0}}\left[w^{(k)}, \hat{w}^{(k)}\right]
$$

with corresponding norm

$$
\left\|\left(w^{\left(-k_{0}\right)}, \ldots, w^{\left(k_{0}\right)}\right)\right\|=\left(\sum_{k=-k_{0}}^{k_{0}}\left[w^{(k)}, w^{(k)}\right]\right)^{1 / 2}
$$

On the spaces $Y_{-k_{0}} \times \ldots \times Y_{k_{0}} \times \mathscr{S}^{3} \times \mathbb{R}^{2}$ and $Y_{-k_{0}} \times \ldots \times Y_{k_{0}} \times \mathscr{S}^{3}$ we use the scalar products

$$
\begin{aligned}
& \quad\left[\left(w^{\left(-k_{0}\right)}, \ldots, z\right),\left(\hat{w}^{\left(-k_{0}\right)}, \ldots, \hat{z}\right)\right] \\
& \left.\quad=\left[\left(w^{\left(-k_{0}\right)}, \ldots, w^{\left(k_{0}\right)}\right),\left(\hat{w}^{\left(-k_{0}\right)}, \ldots, \hat{w}^{\left(k_{0}\right)}\right)\right]+[(s, z), \hat{s}, \hat{z})\right], \\
& \quad\left[\left(w^{\left(-k_{0}\right)}, \ldots, s\right),\left(\hat{w}^{\left(-k_{0}\right)}, \ldots, \hat{s}\right)\right] \\
& \quad=\left[\left(w^{\left(-k_{0}\right)}, \ldots, w^{\left(k_{0}\right)}\right),\left(\hat{w}^{\left(-k_{0}\right)}, \ldots, \hat{w}^{\left(k_{0}\right)}\right)\right]+[s, \hat{s}],
\end{aligned}
$$

where $[(s, z),(\hat{s}, \hat{z})]=\frac{1}{\mathscr{M} b}(s, \hat{s})+(z, \hat{z})$ and $[s, \hat{s}]=\frac{1}{\mathscr{C} b}(s, \hat{s})$, with the norms $\left\|\left(w^{\left(-k_{0}\right)}, \ldots, z\right)\right\|$ and $\left\|\left(w^{\left(-k_{0}\right)}, \ldots, s\right)\right\|$ corresponding to these scalar products.

We also need the operator

$$
(u, \alpha) \rightarrow\left(\begin{array}{cc}
I & 0 \\
0 & -I
\end{array}\right)(u, \alpha)=(u,-\alpha): \mathbb{R}^{3} \times \mathscr{S}^{3} \rightarrow \mathbb{R}^{3} \times \mathscr{S}^{3} .
$$

We now come to the announced definitions. By $\mathscr{H}(\mathbb{R})$ we denote the space of all functions $\left(w^{\left(-k_{0}\right)}, \ldots, w^{\left(k_{0}\right)}, s, z\right): \mathbb{R} \rightarrow Y_{-k_{0}} \times \ldots \times Y_{k_{0}} \times \mathscr{S}^{3} \times \mathbb{R}^{2}$ satisfying

$$
\begin{gathered}
\left(w^{\left(-k_{0}\right)}, \ldots, w^{\left(k_{0}\right)}, s, z\right) \in C([0, L]), \\
w^{(k)} \in C\left(\mathbb{R}, Y_{k}\right) \quad \text { for } \quad k \neq 0, \\
w^{(k)}(x)=\left(\begin{array}{cc}
I & 0 \\
0 & -I
\end{array}\right) w^{(-k)}(-x), \quad x \neq n L \text { for all integers } n, \\
(s, z)(x)=(-s, z)(-x), \quad x \neq n L \text { for all integers } n, \\
\left(w^{\left(-k_{0}\right)}, \ldots, z\right)(x+2 L)=\left(w^{\left(-k_{0}\right)}, \ldots, z\right)(x) .
\end{gathered}
$$

For $T>0$ or $T=\infty$ let $\mathscr{Y}(\mathbb{R} \times[0, T))$ denote the space of all functions

$$
\left(w^{\left(-k_{0}\right)}, \ldots, w^{\left(k_{0}\right)}, s, z\right): \mathbb{R} \times[0, T) \rightarrow Y_{-k_{0}} \times \ldots \times Y_{k_{0}} \times \mathscr{S}^{3} \times \mathbb{R}^{2}
$$


with

$$
\left(w^{\left(-k_{0}\right)}, \ldots, z\right) \in C([0, L] \times[0, T))
$$

and

$$
\left(w^{\left(-k_{0}\right)}, \ldots, z\right)(\cdot, t) \in \mathscr{Y}(\mathbb{R})
$$

for every $t \in[0, T)$.

Lemma 2.3. The spaces $\mathscr{C}(\mathbb{R})$ and $\mathscr{B}(\mathbb{R} \times[0, T)$ ), respectively, are isomorphic to the spaces $\mathscr{Y}(\mathbb{R})$ and $\mathscr{H}(\mathbb{R} \times[0, T))$, respectively, and in both cases the mapping

$$
(w, s, z) \rightarrow\left(\Pi_{-k_{0}} w, \ldots, \Pi_{k_{0}} w, s, z\right)
$$

is an isomorphism with inverse

$$
\left(w^{\left(-k_{0}\right)}, \ldots, w^{\left(k_{0}\right)}, s, z\right) \rightarrow\left(\sum_{k=-k_{0}}^{k_{0}} w^{(k)}, s, z\right)
$$

Proof. We first show that $\mathscr{B}(\mathbb{R})$ and $\mathscr{H}(R)$ are isomorphic. Let the mapping defined in (2.30) be denoted by $\Omega$ and the mapping defined in (2.31) by $\Omega^{\prime}$. From (2.20) and (2.21) it follows that $\Omega^{\prime} \circ \Omega$ is the identity on $\mathscr{B}(\mathbb{R})$ and that $\Omega \circ \Omega^{\prime}$ is the identity on $\mathscr{H}(\mathbb{R})$. To prove the statement it is therefore enough to show that $\Omega(\mathscr{B}(\mathbb{R})) \subset \mathscr{H}(\mathbb{R})$ and $\Omega^{\prime}(\mathscr{Y}(\mathbb{R})) \subset \mathscr{B}(\mathbb{R})$.

To prove the first relation, let $(w, s, z) \in \mathscr{B}(\mathbb{R})$. It must be shown that the function $\left(w^{\left(-k_{0}\right)}, \ldots, w^{\left(k_{0}\right)}, s, z\right)$ with $w^{(k)}=\Pi_{k} w$ satisfies $(2.23)-(2.27)$. This is clear for (2.23), (2.26) and (2.27), which are immediate consequences of (2.3)-(2.5). To prove (2.25), note that the operator $\left(\begin{array}{cc}I & 0 \\ 0 & -I\end{array}\right)$ maps $w_{k}$ to $w_{-k}$ for $2 \leq|k| \leq 4$ and $w_{k}$ to $-w_{k}$ for $|k| \leq 1$ with $w_{k}$ defined in (2.19). This implies

$$
\Pi_{k}\left(\begin{array}{cc}
I & 0 \\
0 & -I
\end{array}\right)=\left(\begin{array}{cc}
I & 0 \\
0 & -I
\end{array}\right) \Pi_{-k} .
$$

Together with (2.4) we thus obtain for $x \notin\{n L: n$ integer $\}$ that

$$
\begin{aligned}
\left(\begin{array}{cc}
I & 0 \\
0 & -I
\end{array}\right) w^{(-k)}(-x) & =\left(\begin{array}{cc}
I & 0 \\
0 & -I
\end{array}\right) \Pi_{-k} w(-x)=\Pi_{k}\left(\begin{array}{cc}
I & 0 \\
0 & -I
\end{array}\right) w(-x) \\
& =\Pi_{k} w(x)=w^{(k)}(x),
\end{aligned}
$$

which is (2.25). To prove (2.24) observe first that (2.23) and (2.25) imply that $w^{(k)}$ is continuous on $[0, L]$ and on $(-L, 0)$. Moreover, note that $w(x)$ has the expansion

$$
w(x)=\sum_{j=-4}^{4} b_{j}(x) w_{j}
$$

with suitable coefficients $b_{j}(x) \in \mathbb{R}$. From (2.3) and (2.6) we conclude with $w(x)=(v(x), \sigma(x)) \in \mathbb{R}^{3} \times \mathscr{S}^{3}$ and $w_{j}=\left(u_{j}, \alpha_{\jmath}\right) \in \mathbb{R}^{3} \times \mathscr{S}^{3}$ that

$$
0=Q \sigma(0+)=Q\left(\sum_{\jmath=-4}^{4} b_{\jmath}(0+) \alpha_{j}\right)=\sum_{j=-4}^{4} b_{j}(0+) Q \alpha_{\jmath} .
$$


From Lemma 2.2 we have $Q \alpha_{-1}=Q \alpha_{0}=Q_{\alpha_{1}}=0$, and (2.15), (2.19) imply $Q \alpha_{\jmath}=-\lambda_{j} r_{|\jmath|-1}$ for $2 \leq|j| \leq 4$. Therefore (2.18) and (2.33) yield

$$
\sum_{j=2}^{4} \sqrt{\mu_{\jmath-1}}\left(b_{-j}(0+)-b_{j}(0+)\right) r_{|\jmath|-1}=0
$$

which yields $b_{j}(0+)=b_{-j}(0+)$ for $|j| \geq 2$. Since

$$
w^{(k)}(x)=\sum_{j \in N_{k}} b_{j}(x) w_{j}
$$

for a suitable set $N_{k}$, which is a subset of $\{j: 2 \leq|j| \leq 4\}$ if $|k| \geq 1$, we obtain with (2.25) and with $N_{-k}=-N_{k}$ that

$$
\begin{aligned}
w^{(k)}(0+) & =\sum_{\jmath \in N_{k}} b_{j}(0+) w_{\jmath}=\sum_{j \in N_{k}} b_{-\jmath}(0+) w_{\jmath} \\
& =\sum_{j \in N_{k}} b_{-j}(0+)\left(\begin{array}{cc}
I & 0 \\
0 & -I
\end{array}\right) w_{-j}=\left(\begin{array}{cc}
I & 0 \\
0 & -I
\end{array}\right) w^{(-k)}(0+)=w^{(k)}(0-),
\end{aligned}
$$

which shows that $w^{(k)}$ is continuous at $x=0$. In the same way it is seen that $w^{(k)}$ is continuous at $x=L$, and therefore everywhere, since $w^{(k)}$ is periodic with period $2 L$. This proves $(2.24)$, whence $\Omega(\mathscr{C}(\mathbb{R})) \subset \mathscr{Y}(\mathbb{R})$.

To prove $\Omega^{\prime}(\mathscr{Y}(\mathbb{R})) \subset \mathscr{C}(\mathbb{R})$ let $\left(w^{\left(-k_{0}\right)}, \ldots, w^{\left(k_{0}\right)}, s, z\right) \in \mathscr{Y}(\mathbb{R})$. It must be shown that $(v, \sigma, s, z)$ with $(v, \sigma)=w=\sum_{k=-k_{0}}^{k_{0}} w^{(k)}$ satisfies (2.3)-(2.6). The relations (2.3) and (2.5) are immediate consequences of (2.23) and (2.27), and (2.4) follows from (2.26) and from (2.25), since for $x \notin\{n L: n$ integer $\}$

$$
w(x)=\sum_{k=-k_{0}}^{k_{0}} w^{(k)}(x)=\sum_{k=-k_{0}}^{k_{0}}\left(\begin{array}{cc}
I & 0 \\
0 & -I
\end{array}\right) w^{(-k)}(-x)=\left(\begin{array}{cc}
I & 0 \\
0 & -I
\end{array}\right) w(-x) .
$$

To prove (2.6), let $w^{(k)}(x)=\left(v^{(k)}(x), \sigma^{(k)}(x)\right)$. Then

$$
Q \sigma(x)=Q \sum_{k=-k_{0}}^{k_{0}} \sigma^{(k)}(x)=\sum_{k=-k_{0}}^{k_{0}} Q \sigma^{(k)}(x)=\sum_{1 \leq|k| \leq k_{0}} Q \sigma^{(k)}(x),
$$

since $\left(v^{(0)}(x), \sigma^{(0)}(x)\right) \in Y_{0}$, and therefore, by Lemma 2.2,

$$
Q \sigma^{(0)}(x)=0 \text {. }
$$

From (2.24) and (2.34) it follows that $Q \sigma$ is continuous on $\mathbb{R}$, which together with (2.4) and (2.5) yields $Q \sigma(0)=Q \sigma(L)=0$, which is (2.6). We thus have $\Omega^{\prime}(\mathscr{Y}(\mathbb{R})) \subset \mathscr{B}(\mathbb{R})$, and it follows that $(2.30)$ defines an isomorphism from $\mathscr{K}(\mathbb{R})$ to $\mathscr{H}(\mathbb{R})$. The corresponding result for $\mathscr{B}(\mathbb{R} \times[0, T))$ and $\mathscr{Y}(\mathbb{R} \times[0, T))$ is an immediate corollary of this result and of the definitions of the spaces $\mathscr{B}(\mathbb{R} \times[0, T))$ and $\mathscr{Y}(\mathbb{R} \times[0, T))$ in $(2.7),(2.8)$ and in (2.28), (2.29). The proof of Lemma 2.3 is complete. 
In the next lemma we write the system of differential equations in characteristic form: Let the operators $J: \mathbb{R}^{3} \times \mathscr{S}^{3} \rightarrow \mathscr{S}^{3}, J^{*}: \mathscr{S}^{3} \rightarrow \mathbb{R}^{3} \times \mathscr{S}^{3}$ be defined by

$$
J w=J(u, \alpha)=\alpha, \quad J^{*} \sigma=(0, \sigma) \in \mathbb{R}^{3} \times \mathscr{S}^{3},
$$

for every $w=(u, \alpha) \in \mathbb{R}^{3} \times \mathscr{S}^{3}, \sigma \in \mathscr{S}^{3}$. Moreover, let

$$
P_{k}=\Pi_{k} J^{*} D P: \mathscr{S}^{3} \rightarrow \mathbb{R}^{3} \times \mathscr{S}^{3}
$$

for $|k| \leq k_{0}$, with $P$ defined in (1.5).

Lemma 2.4. For $(w, s, z) \in \mathscr{B}(\mathbb{R} \times[0, T))$ and $\left(w^{0}, s^{0}, z^{0}\right) \in \mathscr{B}(\mathbb{R})$ let $w^{(k)}(x, t)=$ $\Pi_{k} w(x, t)$ and $w^{0(k)}(x)=\Pi_{k} w^{0}(x)$. Then $(w, s, z)$ is a weak solution of $(2.10)-(2.13)$ to the initial data $\left(w^{0}, s^{0}, z^{0}\right)$ satisfying (2.14) if and only if $\left(w^{\left(-k_{0}\right)}, \ldots, w^{\left(k_{0}\right)}, s, z\right) \in$ $\mathscr{H}(\mathbb{R} \times[0, T))$ is a weak solution of

$$
\begin{gathered}
\partial_{t} w^{(k)}+\lambda^{(k)} \partial_{x} w^{(k)}=-g_{1}\left(|P \sigma-P s|, z_{1}\right)\left(P_{k} \sigma-P_{k} s\right) \\
\partial_{t} s=\mathscr{M} g_{1}\left(|P \sigma-P s|, z_{1}\right)(P \sigma-P s)-\mathscr{l} g_{2}\left(|P s|, z_{2}\right) P s \\
\partial_{t} z=h(z,|P \sigma-P s|,|P s|) \\
\sigma=J \sum_{k=-k_{0}}^{k_{0}} w^{(k)}
\end{gathered}
$$

in $\mathbb{R} \times[0, T)$ with

$$
w^{(k)}(x, 0)=w^{0(k)}(x), \quad s(x, 0)=s^{0}(x), \quad z(x, 0)=z^{0}(x)
$$

for all $x \in \mathbb{R}$ and $-k_{0} \leq k \leq k_{0}$, and with the initial data $\left(w^{0\left(-k_{0}\right)}, \ldots, w^{0\left(k_{0}\right)}, s^{0}, z^{0}\right)$ satisfying

$$
\operatorname{tr} s^{0}(x)=0, \quad x \in \mathbb{R} .
$$

Proof. Equations (2.11) and (2.12) are identical to (2.38) and (2.39). It is clear that (2.13) holds if and only if (2.41) holds. Therefore it is enough to show that (2.10) holds if and only if (2.37) holds. We leave the obvious proof to the reader.

This completes the reformulation of the problem. From Lemma 2.1 and Lemma 2.4 it is clear now that Theorem 1.1 is equivalent to

Theorem 2.5. Let the functions $g_{1}$ and $g_{2}$ satisfy the hypotheses of Theorem 1.1. Then there exist constants $\tilde{M}_{3}, \tilde{M}_{4}>0$ such that to all initial data

$$
W^{0}=\left(w^{0\left(-k_{0}\right)}, \ldots, w^{0\left(k_{0}\right)}, s^{0}, z^{0}\right) \in \mathscr{H}(\mathbb{R})
$$

satisfying

$$
\operatorname{tr} s^{0}(x)=0, \quad x \in \mathbb{R}
$$

there is $T>0$ and a weak solution $W=\left(w^{\left(-k_{0}\right)}, \ldots, w^{\left(k_{0}\right)}, s, z\right) \in \mathscr{Y}(\mathbb{R} \times[0, T))$ of (2.37)-(2.41). This solution satisfies for all $0 \leq t<T$,

$$
\begin{aligned}
& \sup _{-\infty<x<\infty}\left\|\left(w^{\left(-k_{0}\right)}, \ldots, w^{\left(k_{0}\right)}, s\right)(x, t)\right\| \\
& \leq \max \left\{\tilde{M}_{3}\left[1+K^{*}(t, E(0))\right]\left\|\left(w^{0\left(-k_{0}\right)}, \ldots, s^{0}\right)\right\|_{\infty}\right. \\
& \left.\quad\left[\tilde{M}_{4} K^{*}(t, E(0))\left\|\left(w^{0\left(-k_{0}\right)}, \ldots, s^{0}\right)\right\|_{\infty}\right]^{\gamma}\right\}
\end{aligned}
$$


where

$$
\left\|\left(w^{0\left(-k_{0}\right)}, \ldots, s^{0}\right)\right\|_{\infty}=\sup _{0 \leq x \leq L}\left\|\left(w^{0\left(-k_{0}\right)}, \ldots, w^{0\left(k_{0}\right)}, s^{0}\right)(x)\right\| .
$$

The solution $\left(w^{\left(-k_{0}\right)}, \ldots, z\right)$ is locally unique.

Here $E(0), K^{*}(t, E(0))$ and $\gamma$ are the constants from Theorem 1.1. The functions $v^{0}$ and $\sigma^{0}$ in the definition of $E(0)$ are given by

$$
\left(v^{0}, \sigma^{0}\right)=w^{0}=\sum_{k=-k_{0}}^{k_{0}} w^{0(k)} .
$$

Corollary 1.2 is equivalent to

Corollary 2.6. Let the hypothesis of Corollary 1.2 be satisfied. Then to all initial data $W^{0}=\left(w^{\left(-k_{0}\right)}, \ldots, z\right) \in \mathscr{Y}(\mathbb{R})$ satisfying $(2.43)$ there exists a locally unique weak solution $W \in \mathscr{H}(\mathbb{R} \times[0, \infty))$ of $(2.37)-(2.41)$. The solution is contained in $L^{\infty}(\mathbb{R} \times[0, T))$ for every $0<T<\infty$ and satisfies $(2.44)$.

The proofs of Theorem 2.5 and Corollary 2.6 and therefore the proofs of Theorem 1.1 and Corollary 1.2 are given at the end of Sect. 5.

\section{Local Existence and Energy Estimate}

In this section we formulate the local existence theorem and prove an energy estimate, which is needed in the proof of the a-priori $L^{\infty}$-estimate of Theorem 2.5. To formulate the local existence theorem we need some definitions.

For $W \in \mathscr{H}(\mathbb{R}), \hat{W} \in \mathscr{H}(\mathbb{R} \times[0, T))$ and $0 \leq t \leq T$ let

$$
\|W\|_{\infty}=\sup _{x \in \mathbb{R}}\|W(x)\|, \quad\|\hat{W}\|_{\infty, t}=\sup _{\substack{x \in \mathbb{R} \\ 0 \leq \tau<t}}\|\hat{W}(x, \tau)\|,
$$

if the supremum on the right-hand side of the second of these equations is finite. Here $\|\cdot\|$ denotes the norm on $Y_{-k_{0}} \times \ldots \times Y_{k_{0}} \times \mathscr{S}^{3} \times \mathbb{R}^{2}$ defined after Lemma 2.2.

Let $T>0$ and $W \in \mathscr{H}(\mathbb{R} \times[0, T))$ be a weak solution of (2.37)-(2.41). We call $T$ maximal time of existence of $W$ if and only if there does not exist $\delta>0$ and a weak solution $\hat{W} \in \mathscr{Y}(\mathbb{R} \times[0, T+\delta))$ of (2.37)-(2.41) with $\hat{W}_{\mid \mathbb{R} \times[0, T)}=W$.

The local existence theorem is

Theorem 3.1. Let the functions $g_{1}, g_{2}: \mathbb{R}_{0}^{+} \times \mathbb{R} \rightarrow \mathbb{R}_{0}^{+}, h: \mathbb{R}^{2} \times\left(\mathbb{R}_{0}^{+}\right)^{2} \rightarrow \mathbb{R}^{2}$ be locally Lipschitz continuous. Then to every initial data $W^{0}=\left(w^{0\left(-k_{0}\right)}, \ldots, w^{0\left(k_{0}\right)}, s^{0}, z^{0}\right) \in$ $\mathscr{H}(\mathbb{R})$ satisfying $\operatorname{tr} s^{0}(x)=0$ for all $x \in \mathbb{R}$ there exists $a T>0$ and a weak solution $W=\left(w^{\left(-k_{0}\right)}, \ldots, w^{\left(k_{0}\right)}, s, z\right) \in \mathscr{H}(\mathbb{R} \times[0, T))$ of (2.37)-(2.41). The solution is locally unique.

If there exists a maximal time of existence $T_{\infty}<\infty$ of this solution, then

$$
\sup _{T<T_{\infty}}\|W\|_{\infty, T}=\infty .
$$

Sketch of the Proof. We only state the idea of the standard proof. Let $T>0$. Below we define an operator $B: \mathscr{Y}(\mathbb{R} \times[0, T)) \rightarrow \mathscr{H}(\mathbb{R} \times[0, T))$ such that $W$ is a fixed point of $B$ if and only if $W$ is a weak solution of (2.37)-(2.41). Of course $B$ depends on $W^{0}$. Next it is shown that if $C>0$ is a constant, then there exists $T_{0}=T_{0}(C)>0$ 
such that to all initial data $W^{0}$ with $\left\|W^{0}\right\|_{\infty} \leq C / 2$ the operator $B$ maps the closed set of all $W \in \mathscr{Y}\left(\mathbb{R} \times\left[0, T_{0}\right)\right)$ with $\|W\|_{\infty, T_{0}} \leq C$ into itself and is a contraction on this set, hence has a unique fixed point. This yields existence and local uniqueness of the solution, and at the same time (3.1), in a well known way. To define $B$ let $W=\left(w^{\left(-k_{0}\right)}, \ldots, z\right) \in \mathscr{Y}(\mathbb{R} \times[0, T))$ and set

$$
\begin{aligned}
\hat{w}^{(k)}(x, t)= & \int_{0}^{t}\left[-g_{1}\left(|P \sigma-P s|, z_{1}\right)\left(P_{k} \sigma-P_{k} s\right)\right]\left(x+\lambda^{(k)}(\tau-t), \tau\right) d \tau \\
& +w^{0(k)}\left(x-\lambda^{(k)} t\right) \\
\hat{s}(x, t)= & \mathscr{M b} \int_{0}^{t}\left[g_{1}\left(|P \sigma-P s|, z_{1}\right)(P \sigma-P s)\right. \\
& \left.-g_{2}\left(|P s|, z_{2}\right) P s\right](x, \tau) d \tau+s^{0}(x), \\
\hat{z}(x, t)= & \int_{0}^{t}\left[h(z,|P \sigma-P s|,|P s|](x, \tau) d \tau+z^{0}(x)\right.
\end{aligned}
$$

with

$$
\sigma=J \sum_{k=-k_{0}}^{k_{0}} w^{(k)}
$$

Now set

$$
B W=\left(\hat{w}^{\left(-k_{0}\right)}, \ldots, \hat{w}^{\left(k_{0}\right)}, \hat{s}, \hat{z}\right)
$$

The details of the proof are left to the reader.

Next we state the energy inequality. For $W=\left(w^{\left(-k_{0}\right)}, \ldots, w^{\left(k_{0}\right)}, s, z\right) \in \mathscr{H}(\mathbb{R} \times$ $[0, T))$ define the energy

$$
\begin{aligned}
E(t) & =E(t, W)=\frac{1}{2} \int_{0}^{L} \sum_{k=-k_{0}}^{k_{0}}\left[w^{(k)}(x, t), w^{(k)}(x, t)\right]+\frac{1}{\mathscr{M} b}|s(x, t)|^{2} d x \\
& =\frac{1}{2} \int_{0}^{L}[w(x, t), w(x, t)]+\frac{1}{\mathscr{M} b}|s(x, t)|^{2} d x \\
& =\frac{1}{2} \int_{0}^{L}|v(x, t)|^{2}+\left(D^{-1} \sigma(x, t), \sigma(x, t)\right)+\frac{1}{\mathscr{M b}}|s(x, t)|^{2} d x
\end{aligned}
$$

with

$$
w=(v, \sigma)=\sum_{k=-k_{0}}^{k_{0}} w^{(k)}
$$


Lemma 3.2. If $W \in \mathscr{Y}(\mathbb{R} \times[0, T))$ is a weak solution of (2.37)-(2.41), then

$$
\begin{aligned}
E(t)-E(0)= & -\int_{0}^{t} \int_{0}^{L} g_{1}\left(|P \sigma-P s|, z_{1}\right)|P \sigma-P s|^{2} d x d \tau \\
& -\int_{0}^{t} \int_{0}^{L} g_{2}\left(|P s|, z_{2}\right)|P s|^{2} d x d \tau .
\end{aligned}
$$

Proof. Since

$$
-g_{1}\left(|P \sigma-P s|, z_{1}\right)\left(P_{k} \sigma-P_{k} s\right) \in C([0, L] \times[0, T))
$$

it follows from (2.37) that $w^{(k)}(x, t)$ is continuously differentiable in direction of the vector $\left(\lambda^{(k)}, 1\right)$. We denote the derivative by $D_{\left(\lambda^{(k)}, 1\right)} w^{(k)}$ and obtain from the fundamental theorem of calculus

$$
\begin{aligned}
& 2 \sum_{k=-k_{0}}^{k_{0}} \int_{0}^{t} \int_{0}^{L}\left[w^{(k)}, D_{\left(\lambda^{(k)}, 1\right)} w^{(k)}\right] d x d \tau \\
& =\sum_{k=-k_{0}}^{k_{0}} \int_{0}^{t} \int_{0}^{L} D_{\left(\lambda^{(k)}, 1\right)}\left[w^{(k)}, w^{(k)}\right] d x d \tau \\
& =\sum_{k=-k_{0}}^{k_{0}} \int_{0}^{L}\left[w^{(k)}(x, t), w^{(k)}(x, t)\right]-\left[w^{(k)}(x, 0), w^{(k)}(x, 0)\right] d x \\
& \left.\quad+\lambda^{(k)} \int_{0}^{t}\left[w^{(k)}(L, \tau), w^{(k)}(L, \tau)\right]-\left[w^{(k)}(0, \tau), w^{(k)}(0, \tau)\right] d \tau\right\}
\end{aligned}
$$

For $k \neq 0$ the function $x \mapsto w^{(k)}(x, t)$ is continuous on $\mathbb{R}$,by (2.24), and therefore satisfies (2.25) for all $x \in \mathbb{R}$. From (2.25) and (2.27) we thus obtain for $k \neq 0$ and $x=0$ or $x=L$

$$
w^{(k)}(x, t)=\left(\begin{array}{cc}
I & 0 \\
0 & -I
\end{array}\right) w^{(-k)}(x, t)
$$

hence

$$
\left[w^{(k)}(x, t), w^{(k)}(x, t)\right]=\left[w^{-k}(x, t), w^{-k}(x, t)\right]
$$

Since $\lambda^{(0)}=0$ and $\lambda^{(k)}=-\lambda^{(-k)}$, we thus obtain

$$
\sum_{k=-k_{0}}^{k_{0}} \lambda^{(k)} \int_{0}^{t}\left[w^{(k)}(L, \tau), w^{(k)}(L, \tau)\right]-\left[w^{(k)}(0, \tau), w^{(k)}(0, \tau)\right] d \tau=0
$$


Insertion of this equation into (3.7) yields together with (2.37)

$$
\begin{aligned}
& \int_{0}^{L}[w(x, t), w(x, t)]-[w(x, 0), w(x, 0)] d x \\
& \quad=-2 \sum_{k=-k_{0}}^{k_{0}} \int_{0}^{t} \int_{0}^{L}\left[w^{(k)}, g_{1}\left(|P \sigma-P s|, z_{1}\right)\left(P_{k} \sigma-P_{k} s\right)\right] d x d \tau \\
& =-2 \int_{0}^{t} \int_{0}^{L} g_{1}\left(|P \sigma-P s|, z_{1}\right)(P \sigma-P s, P \sigma) d x d \tau
\end{aligned}
$$

because the definition of $P_{k}$ in (2.36) implies

$$
\begin{aligned}
& \sum_{k=-k_{0}}^{k_{0}}\left[w^{(k)}(x, t), g_{1}\left(|P \sigma-P s|, z_{1}\right)\left(P_{k} \sigma-P_{k} s\right)(x, t)\right] \\
& \quad=\sum_{k=-k_{0}}^{k_{0}} g_{1}\left(|P \sigma-P s|, z_{1}\right)\left[w^{(k)}, J^{*} D P(\sigma-s)\right] \\
& \quad=g_{1}\left(|P \sigma-P s|, z_{1}\right)\left[w, J^{*} D P(\sigma-s)\right] \\
& \quad=g_{1}\left(|P \sigma-P s|, z_{1}\right)\left(D^{-1} J w, D(P \sigma-P s)\right) \\
& \quad=g_{1}\left(|P \sigma-P s|, z_{1}\right)(\sigma, P \sigma-P s)=g_{1}\left(|P \sigma-P s|, z_{1}\right)(P \sigma, P \sigma-P s) .
\end{aligned}
$$

Finally, (2.38) yields

$$
\begin{aligned}
& \frac{1}{\mathscr{M} b} \int_{0}^{L}|s(x, t)|^{2}-|s(x, 0)|^{2} d x \\
& =\frac{2}{\mathscr{M l}} \int_{0}^{t} \int_{0}^{L}\left(s_{t}(x, \tau), s(x, \tau)\right) d x d \tau \\
& =2 \int_{0}^{t} \int_{0}^{L}\left(g_{1}\left(|P \sigma-P s|, z_{1}\right)(P \sigma-P s)-g_{2}\left(|P s|, z_{2}\right) P s, P s\right) d x d \tau .
\end{aligned}
$$

Combination of this inequality with (3.8) yields the statement of the lemma.

\section{Fundamental Solutions}

In the proof of Theorem 2.5 we also need some information about fundamental solutions of linearized versions of Eq. (2.37) and (2.38). These fundamental solutions are defined and studied in this section. As motivation for the following definitions note that Eq. (2.37) and (2.38) can be written in the form

$$
\frac{\partial}{\partial \tau} w^{(k)}\left(x+\lambda^{(k)}(\tau-t), \tau\right)=-g_{1} P_{k} J w^{(k)}\left(x+\lambda^{(k)}(\tau-t), \tau\right)-a
$$


for $k \neq 0$, and

$$
\frac{\partial}{\partial \tau}\left(\begin{array}{c}
w^{(0)}(x, \tau) \\
s(x, \tau)
\end{array}\right)=\left(\begin{array}{cc}
-g_{1} P_{0} J & g_{1} P_{0} \\
\mathscr{M} g_{1} P J & -\mathscr{M}\left(g_{1}+g_{2}\right) P
\end{array}\right)\left(\begin{array}{c}
w^{(0)}(x, \tau) \\
s(x, \tau)
\end{array}\right)-b
$$

with

$$
a=g_{1} P_{k}\left(J \sum_{\substack{j=-k_{0} \\
j \neq k}}^{k_{0}} w^{(j)}-s\right), \quad b=g_{1}\left(\begin{array}{c}
P_{0} \\
-\mathscr{L} P
\end{array}\right) J \sum_{\substack{j=-k_{0} \\
j \neq 0}}^{k_{0}} w^{(j)} .
$$

If $g_{1}\left(|P \sigma-P s|, z_{1}\right), g_{2}\left(|P s|, z_{2}\right), a$ and $b$ were known, then (4.1) would be a linear system of ordinary differential equations for $w^{(k)}$ and (4.2) would be a linear system of ordinary differential equations for the pair $\left(w^{(0)}, s\right)$. The functions $F_{k}(\tau ; x, t)$ defined in the following essentially are the fundamental solutions of these systems, and in the proof of the a-priori estimates in the succeeding section they are used to represent the solutions $w^{(k)}$ and $s$.

A linear operator $F$ from $\mathbb{R}^{3} \times \mathscr{Y}^{3}$ to the subspace $Y_{k}$ of $\mathbb{R}^{3} \times \mathscr{S}^{3}$ can be represented by a matrix. If this operator depends on a real parameter $\tau$, then $\frac{d}{d \tau} F(\tau)$ denotes the operator obtained by differentiation of the matrix elements of $F$. In this section we assume that $\left(\tilde{w}^{\left(-k_{0}\right)}, \ldots, \tilde{w}^{\left(k_{0}\right)}, \tilde{s}, \tilde{z}\right) \in \mathscr{H}(\mathbb{R} \times[0, T))$ is a given function. For brevity we set

$$
\begin{aligned}
& \tilde{g}_{1}(x, t)=g_{1}\left(|P \tilde{\sigma}(x, t)-P \tilde{s}(x, t)|, \tilde{z}_{1}(x, t)\right) \\
& \tilde{g}_{2}(x, t)=g_{2}\left(|P \tilde{s}(x, t)|, \tilde{z}_{2}(x, t)\right)
\end{aligned}
$$

with $\tilde{w}=(\tilde{v}, \tilde{\sigma})=\sum_{k=-k_{0}}^{k_{0}} \tilde{w}^{(k)}$.

Definition 4.1. Let $(x, t) \in \mathbb{R} \times[0, T), 0 \leq t_{0}, \tau<T$.

(i) For every integer $k \neq 0$ with $|k| \leq k_{0}$ let

$$
\hat{F}_{k}(\tau)=\hat{F}_{k}\left(t_{0}, \tau ; x, t\right)
$$

be the linear operator from $\mathbb{R}^{3} \times \mathscr{S}^{3}$ to $Y_{k}$ such that

$$
\begin{aligned}
\frac{\partial}{\partial \tau} \hat{F}_{k}(\tau) & =-\tilde{g}_{1}\left(x+\lambda^{(k)}(\tau-t), \tau\right) P_{k} J \hat{F}_{k}(\tau), \quad 0 \leq \tau<T \\
\hat{F}_{k}\left(t_{0}\right) & =\Pi_{k} .
\end{aligned}
$$

(ii) Let

$$
\hat{F}_{0,1}(\tau)=\hat{F}_{0,1}\left(t_{0}, \tau ; x, t\right)
$$

be the linear operator from $\mathbb{R}^{3} \times \mathscr{P}^{3} \times \mathscr{S}^{3}$ to $Y_{0}$ and

$$
\hat{F}_{0,2}(\tau)=\hat{F}_{0,2}\left(t_{0}, \tau ; x, t\right)
$$

be the linear operator from $\mathbb{R}^{3} \times \mathscr{S}^{3} \times \mathscr{S}^{3}$ to $\mathscr{S}^{3}$ such that

$$
\begin{aligned}
& \frac{\partial}{\partial \tau}\left(\begin{array}{c}
\hat{F}_{0,1}(\tau) \\
\hat{F}_{0,2}(\tau)
\end{array}\right) \\
& =\left(\begin{array}{cc}
-\tilde{g}_{1}(x, \tau) P_{0} J & \tilde{g}_{1}(x, \tau) P_{0} \\
\mathscr{M} \tilde{g}_{1}(x, \tau) P J & -\mathscr{M b}\left(\tilde{g}_{1}(x, \tau)+\tilde{g}_{2}(x, \tau)\right) P
\end{array}\right)\left(\begin{array}{l}
\hat{F}_{0,1}(\tau) \\
\hat{F}_{0,2}(\tau)
\end{array}\right)
\end{aligned}
$$


for $0 \leq \tau<T$, and

$$
\left(\begin{array}{c}
\hat{F}_{0,1}\left(t_{0}\right) \\
\hat{F}_{0,2}\left(t_{0}\right)
\end{array}\right)=\Pi_{0} \oplus I
$$

with $\Pi_{0} \oplus I: \mathbb{R}^{3} \times \mathscr{S}^{3} \times \mathscr{S}^{3} \rightarrow Y_{0} \times \mathscr{S}^{3}$ defined by

$$
\left(\Pi_{0} \oplus I\right)(v, \sigma, s)=\left(\Pi_{0}(v, \sigma), s\right) .
$$

We set

$$
\hat{F}_{0}(\tau)=\hat{F}_{0}\left(t_{0}, \tau ; x, t\right)=\left(\begin{array}{c}
\hat{F}_{0,1}(\tau) \\
\hat{F}_{0,2}(\tau)
\end{array}\right) .
$$

$\hat{F}_{0}(\tau)$ is a linear operator from $\mathbb{R}^{3} \times \mathscr{S}^{3} \times \mathscr{S}^{3}$ to $Y_{0} \times \mathscr{S}^{3}$.

(iii) For every integer $k$ with $|k| \leq k_{0}$ let

$$
F_{k}\left(t_{0} ; x, t\right)=\hat{F}_{k}\left(t_{0}, t ; x, t\right) .
$$

To study the properties of $F_{k}$ we need the following results.

Lemma 4.2. (i) For every integer $k$ with $|k| \leq k_{0}$ the operator $P_{k} J_{\mid Y_{k}}: Y_{k} \rightarrow Y_{k}$ is selfadjoint and nonnegative, if the space $Y_{k}$ is equipped with the scalar product [w, $\left.\hat{w}\right]$ induced by the scalar product on $\mathbb{R}^{3} \times \mathscr{S}^{3}$.

(ii) The operators $U_{1}, U_{2}: Y_{0} \times \mathscr{S}^{3} \rightarrow Y_{0} \times \mathscr{S}^{3}$ defined by

$$
U_{1}=\left(\begin{array}{cc}
-P_{0} J & P_{0} \\
\mathscr{M} P J & -\mathscr{M b P}
\end{array}\right), \quad U_{2}=\left(\begin{array}{cc}
0 & 0 \\
0 & -\mathscr{M b} P
\end{array}\right)
$$

are selfadjoint and nonpositive, if the space $Y_{0} \times \mathscr{S}^{3}$ is equipped with the scalar product $[(w, s),(\hat{w}, \hat{s})]$ induced by the scalar product on $\mathbb{R}^{3} \times \mathscr{S}^{3} \times \mathscr{S}^{3}$. For every $(x, t) \in \mathbb{R} \times[0, T)$ the operator

$$
U=\tilde{g}_{1}(x, t) U_{1}+\tilde{g}_{2}(x, t) U_{2}: Y_{0} \times \mathscr{S}^{3} \rightarrow Y_{0} \times \mathscr{S}^{3}
$$

is selfadjoint and nonpositive.

Proof. (i) The definitions of $[w, \hat{w}]$ at the beginning of Sect. 2 and of $J, J^{*}, P_{k}$ in (2.35), (2.36) imply for $w \in \mathbb{R}^{3} \times \mathscr{S}^{3}, \hat{w} \in Y_{k}$

$$
\begin{aligned}
{\left[P_{k} J w, \hat{w}\right] } & =\left[\Pi_{k} J^{*} D P J w, \hat{w}\right]=\left[J^{*} D P J w, \Pi_{k} \hat{w}\right] \\
& =\left[J^{*} D P J w, \hat{w}\right]=\left(D^{-1} D P J w, J \hat{w}\right)=(P J w, P J \hat{w}) .
\end{aligned}
$$

If $w, \hat{w} \in Y_{k}$ we obtain in the same way

$$
\left[w, P_{k} J \hat{w} P_{k} \hat{w}\right]=(P J w, P J \hat{w}) .
$$

Equations (4.10) and (4.11) together show that $P_{k} J_{\mid Y_{k}}$ is selfadjoint and nonnegative. (ii) Equation (4.10) and the definition of the scalar product on $\mathbb{R}^{3} \times \mathscr{S}^{3} \times \mathscr{S}^{3}$ at the beginning of Sect. 2 yields for $(w, s) \in \mathbb{R}^{3} \times \mathscr{S}^{3} \times \mathscr{S}^{3},(\hat{w}, \hat{s}) \in Y_{0} \times \mathscr{S}^{3}$

$$
\begin{aligned}
{\left[U_{1}(w, s),(\hat{w}, \hat{s})\right] } & =-\left[P_{0} J w, \hat{w}\right]+\left[P_{0} s, \hat{w}\right]+\frac{1}{\mathscr{C}}(\mathscr{M} P J w, \hat{s})-\frac{1}{\mathscr{C} b}(\mathscr{M} P s, \hat{s}) \\
& =-(P J w, P J \hat{w})+\left[\Pi_{0} J^{*} D P s, \hat{w}\right]+(P J w, P \hat{s})-(P s, P \hat{s}) \\
& =-(P J w, P(J \hat{w}-\hat{s}))+\left(D^{-1} D P s, J \hat{w}\right)-(P s, P \hat{s}) \\
& =-(P(J w-s), P(J \hat{w}-\hat{s})) .
\end{aligned}
$$


For $(w, s),(\hat{w}, \hat{s}) \in Y_{0} \times \mathscr{S}^{3}$ we obtain in the same way

$$
\left[(w, s), U_{1}(\hat{w}, \hat{s})\right]=-(P(J w-s), P(J \hat{w}-\hat{s})) .
$$

This equation and (4.12) together show that $U_{1}$ is selfadjoint and nonpositive. To prove this statement for $U_{2}$, note that for $(w, s),(\hat{w}, \hat{s}) \in \mathbb{R}^{3} \times \mathscr{S}^{3} \times \mathscr{S}^{3}$ we obtain

$$
\left[U_{2}(w, s),(\hat{w}, \hat{s})\right]=\frac{1}{\mathscr{M} b}(-\mathscr{M b} P s, \hat{s})=-(P s, P \hat{s})=\left[(w, s), U_{2}(\hat{w}, \hat{s})\right]
$$

and the statement follows. The statement for $U$ is an immediate consequence of these results, since $\tilde{g}_{1}(x, t), \tilde{g}_{2}(x, t) \geq 0$.

After this preparation we can prove the following

Lemma 4.3. (i) There exists $\mu>0$ such that for every integer $k \neq 0$ with $|k| \leq k_{0}$, for all $(x, t) \in \mathbb{R} \times[0, T), 0 \leq t_{0} \leq t$ and all $w \in \mathbb{R}^{3} \times \mathscr{S}^{3}$

$$
\left\|F_{k}\left(t_{0} ; x, t\right) w\right\| \leq\left\|\Pi_{k} w\right\| \leq\|w\|
$$

and

$$
\left\|F_{k}\left(t_{0} ; x, t\right) P_{k} J w\right\| \leq \exp \left\{-\mu \int_{t_{0}}^{t} \tilde{g}_{1}\left(x+\lambda^{(k)}(\tau-t), \tau\right) d \tau\right\}\left\|P_{k} J w\right\|
$$

with $\|w\|=[w, w]^{1 / 2}$.

(ii) Let $(x, t) \in \mathbb{R} \times[0, T), 0 \leq t_{0} \leq t$ and $(w, s) \in \mathbb{R}^{3} \times \mathscr{S}^{3} \times \mathscr{S}^{3}$. Then

$$
\left\|\left[F_{0}\left(t_{0} ; x, t\right)\right](w, s)\right\| \leq\left\|\left[\Pi_{0} \oplus I\right](w, s)\right\| \leq\|(w, s)\|
$$

with $\|(w, s)\|=[(w, s),(w, s)]^{1 / 2}$.

(iii) Let $g_{2}(\eta, \zeta)=0$ for all $(\eta, \zeta) \in \mathbb{R}_{0}^{+} \times \mathbb{R}$. Then there exists $\nu>0$ such that for all $(x, t) \in \mathbb{R} \times[0, T), 0 \leq t_{0} \leq t$ and $w \in \mathbb{R}^{3} \times \mathscr{S}^{3}$

$$
\left\|F_{0}\left(t_{0} ; x, t\right)\left(\begin{array}{c}
-P_{0} \\
\mathscr{l} P P
\end{array}\right) J w\right\| \leq \exp \left\{-\nu \int_{t_{0}}^{t} \tilde{g}_{1}(x, \tau) d \tau\right\}\left\|\left(\begin{array}{c}
-P_{0} \\
\mathscr{M} P
\end{array}\right) J w\right\|,
$$

where the operator $\left(\begin{array}{c}-P_{0} \\ \mathscr{M} P\end{array}\right): \mathscr{S}^{3} \rightarrow Y_{0} \times \mathscr{S}^{3}$ is defined by

$$
\left(\begin{array}{c}
-P_{0} \\
\mathscr{M} P
\end{array}\right) s=\left(-P_{0} s, \mathscr{M} P s\right) .
$$

Proof. (i) Since $P_{k} J_{\mid Y_{k}}$ is selfadjoint and nonnegative on $Y_{k}$, there exists a set of eigenvalues $0 \leq \mu_{1}^{(k)} \leq \mu_{2}^{(k)} \leq \ldots \leq \mu_{l_{k}}^{(k)}$ of this operator, counted according to multiplicity, and an orthonormal system of eigenvectors $\omega_{1}^{(k)}, \ldots, \omega_{l_{k}}^{(k)}$, which is complete in $Y_{k}$. By

$$
\hat{\Pi}_{\omega_{j}^{(k)}}: \mathbb{R}^{3} \times \mathscr{S}^{3} \rightarrow \mathbb{R}^{3} \times \mathscr{P}^{3}
$$

we denote the orthogonal projector onto the subspace spanned by the eigenvector $\omega_{j}^{(k)}$. With these definitions and with Definition 4.1 we obtain

$$
\hat{F}_{k}\left(t_{0}, \tau ; x, t\right)=\sum_{j=1}^{l_{k}}\left(\exp \left\{-\mu_{j}^{(k)} \int_{t_{0}}^{\tau} \tilde{g}_{1}\left(x+\lambda^{(k)}(\eta-t), \eta\right) d \eta\right\} \hat{\Pi}_{\omega_{j}^{(k)}}\right) .
$$


To prove this, note that differentiation of this function shows that (4.4) is satisfied, and that

$$
\hat{F}_{k}\left(t_{0}, t_{0} ; x, t\right)=\sum_{j=1}^{l_{k}} \hat{\Pi}_{\omega_{j}^{(k)}}=\Pi_{k}
$$

which is (4.5). This yields the statements, since the solution of (4.4), (4.5) is unique. For the function defined in (4.9) we thus have

$$
F_{k}\left(t_{0} ; x, t\right)=\sum_{\jmath=1}^{l_{k}}\left(\exp \left\{-\mu_{\jmath}^{(k)} \int_{t_{0}}^{t} \tilde{g}_{1}\left(x+\lambda^{(k)}(\eta-t), \eta\right) d \eta\right\} \hat{\Pi}_{\omega_{\jmath}^{(k)}}\right)
$$

whence

$$
\left\|F_{k}\left(t_{0} ; x, t\right) w\right\|^{2} \leq \sum_{\jmath=1}^{l_{k}}\left\|\hat{\Pi}_{\omega_{\jmath}^{(k)}} w\right\|^{2}=\left\|\Pi_{k} w\right\|^{2} \leq\|w\|^{2}
$$

for $0 \leq t_{0} \leq t$ and $w \in \mathbb{R}^{3} \times \mathscr{Y}^{3}$. This proves (4.14).

Observe now that for $w \in \operatorname{ker}\left(P_{k} J\right) \cap Y_{k}$ Eq. (4.10) yields

$$
0=\left[P_{k} J w, w\right]=(P J w, P J w),
$$

hence $P J w=0$. From (2.36) we thus obtain

$$
\operatorname{ker}\left(P_{k} J\right) Y_{k}=\operatorname{ker}(P J) \cap Y_{k} .
$$

Now if $\mu_{\jmath}^{(k)}=0$, then $\hat{\Pi}_{\omega_{\jmath}^{(k)}}$ is a projector onto a subspace of $\operatorname{ker}\left(P_{k} J\right) \cap Y_{k}$. Therefore Eqs. (4.10) and (4.19) yield for all $w, \hat{w} \in \mathbb{R}^{3} \times \mathscr{S}^{3}$,

$$
\left[\hat{\Pi}_{\omega_{\jmath}^{(k)}} P_{k} J w, \hat{w}\right]=\left[P_{k} J w, \hat{\Pi}_{\omega_{j}^{(k)}} \hat{w}\right]=\left(P J w, P J \hat{\Pi}_{\omega_{j}^{(k)}} \hat{w}\right)=0,
$$

which implies $\hat{\Pi}_{\omega_{\jmath}^{(k)}} P_{k} J=0$. With

$$
\mu=\min \left(\left\{\mu_{\imath}^{(j)}:-k_{0} \leq j \leq k_{0}, 1 \leq i \leq l_{j}, \mu_{\imath}^{(j)} \neq 0\right\} \cup\{1\}\right)>0,
$$

we thus obtain from (4.18) that

$$
\begin{aligned}
& \left\|\left[F_{k}\left(t_{0} ; x, t\right)\right] P_{k} J w\right\|^{2} \\
& =\sum_{\substack{j=1 \\
\mu_{j}^{(k)} \neq 0}}^{l_{k}}\left(\exp \left\{-\mu_{\jmath}^{(k)} \int_{t_{0}}^{t} \tilde{g}_{1}\left(x+\lambda^{(k)}(\eta-t), \eta\right) d \eta\right\}\right)^{2}\left\|\hat{\Pi}_{\omega_{\jmath}^{(k)}} P_{k} J w\right\|^{2} \\
& \quad \leq\left(\exp \left\{-\mu \int_{t_{0}}^{t} \tilde{g}_{1}\left(x+\lambda^{(k)}(\eta-t), \eta\right) d \eta\right\}\right)^{2}\left\|P_{k} J w\right\|^{2},
\end{aligned}
$$

which proves (4.15).

(ii) From (4.6), (4.8) and the definition of $U$ in Lemma 4.2 we obtain with $u=(w, s)$,

$$
\begin{aligned}
\frac{d}{d \tau}\left\|\hat{F}_{0}(\tau) u\right\|^{2} & =2\left[\frac{d}{d \tau} \hat{F}_{0}(\tau) u, \hat{F}_{0}(\tau) u\right] \\
& =2\left[U \hat{F}_{0}(\tau) u, \hat{F}_{0}(\tau) u\right] \leq 0,
\end{aligned}
$$


since $\hat{F}_{0}(\tau) u \in Y_{0} \times \mathscr{S}^{3}$ and $U$ is nonpositive on this space, by Lemma 4.2(ii). For $t \geq t_{0}$ we thus conclude from (4.7) that

$$
\begin{aligned}
\left\|F_{0}\left(t_{0} ; x, t\right) u\right\| & =\left\|\hat{F}_{0}\left(t_{0}, t ; x, t\right) u\right\| \\
& \leq\left\|\hat{F}_{0}\left(t_{0}, t_{0} ; x, t\right) u\right\|=\left\|\left[\Pi_{0} \oplus I\right] u\right\| \leq\|u\|,
\end{aligned}
$$

which is (4.16).

(iii) Since $U_{1}$ is selfadjoint and nonpositive on $Y_{0} \times \mathscr{S}^{3}$, there exists a set of eigenvalues $0 \geq \nu_{1} \geq \ldots \geq \nu_{l}$ of $U_{1}$, counted according to multiplicity, and an orthonormal system of eigenvectors $\vartheta_{1}, \ldots, \vartheta_{l}$, complete in $Y_{0} \times \mathscr{S}^{3}$. By

$$
\tilde{\Pi}_{\vartheta_{\jmath}}: \mathbb{R}^{3} \times \mathscr{S}^{3} \times \mathscr{S}^{3} \rightarrow \mathbb{R}^{3} \times \mathscr{S}^{3} \times \mathscr{S}^{3}
$$

we denote the orthogonal projector onto the space spanned by the eigenvector $\vartheta_{\jmath}$. When $g_{2} \equiv 0$, Eqs. (4.6) and (4.7) can be written as

$$
\frac{\partial}{\partial \tau} \hat{F}_{0}(\tau)=\tilde{g}_{1}(x, t) U_{1} \hat{F}_{0}(\tau), \quad \hat{F}_{0}\left(t_{0}\right)=\Pi_{0} \oplus I
$$

From these equations we obtain exactly as in the proof of (4.18) that

$$
F_{0}\left(t_{0} ; x, t\right)=\sum_{j=1}^{l}\left(\exp \left\{\nu_{j} \int_{t_{0}}^{t} \tilde{g}_{1}(x, \tau) d \tau\right\} \tilde{\Pi}_{\vartheta_{\jmath}}\right) \text {. }
$$

Now observe that (4.12) yields for $(w, s) \in\left(\operatorname{ker} U_{1}\right) \cap\left(Y_{0} \times \mathscr{S}^{3}\right)$ that

$$
0=\left[U_{1}(w, s),(w, s)\right]=-(P(J w-s), P(J w-s)),
$$

hence $P(J w-s)=0$. Together with the definition of $U_{1}$ in Lemma 4.2 and the definition of $P_{0}$ in (2.36) it follows that

$$
\left(\operatorname{ker} U_{1}\right) \cap\left(Y_{0} \times \mathscr{S}^{3}\right)=\left\{(w, s) \in Y_{0} \times \mathscr{S}^{3}: P(J w-s)=0\right\} .
$$

Now if $\nu_{\jmath}=0$, then $\tilde{\Pi}_{\vartheta_{\jmath}}$ is a projector onto a subspace of $\left(\operatorname{ker} U_{1}\right) \cap\left(Y_{0} \times \mathscr{S}^{3}\right)$. Therefore (4.12) and (4.21) imply for $(w, s),(\hat{w}, \hat{s}) \in \mathbb{R}^{3} \times \mathscr{S}^{3} \times \mathscr{S}^{3}$ with

$$
\left(w^{\prime}, s^{\prime}\right)=\tilde{\Pi}_{\vartheta_{\jmath}}(\hat{w}, \hat{s}) \in\left(\operatorname{ker} U_{1}\right) \cap\left(Y_{0} \times \mathscr{S}^{3}\right)
$$

that

$$
\begin{aligned}
{\left[\tilde{\Pi}_{\vartheta_{j}} U_{1}(w, s),(\hat{w}, \hat{s})\right] } & =\left[U_{1}(w, s), \tilde{\Pi}_{\vartheta_{\jmath}}(\hat{w}, \hat{s})\right] \\
& =-\left(P(J w-s), P\left(J w^{\prime}-s^{\prime}\right)\right)=0
\end{aligned}
$$

which yields $\tilde{\Pi}_{\vartheta_{\jmath}} U_{1}=0$. Again using the definition of $U_{1}$ in Lemma 4.2 we thus conclude that

$$
\tilde{\Pi}_{\vartheta_{j}}\left(\begin{array}{c}
-P_{0} \\
\mathscr{M} P
\end{array}\right) J w=\tilde{\Pi}_{\vartheta_{\jmath}} U_{1}(w, 0)=0
$$

for all $w \in \mathbb{R}^{3} \times \mathscr{S}^{3}$. With

$$
\nu=\min \left(\left\{-\nu_{j}: 1 \leq j \leq l, \nu_{j} \neq 0\right\} \cup\{1\}\right)>0,
$$


we thus conclude from (4.20) that

$$
\begin{aligned}
& \left\|F_{0}\left(t_{0} ; x, t\right)\left(\begin{array}{c}
-P_{0} \\
\mathscr{C O P}
\end{array}\right) J w\right\|^{2} \\
& =\sum_{\substack{j=1 \\
\nu_{\jmath} \neq 0}}^{l}\left(\exp \left\{\nu_{\jmath} \int_{t_{0}}^{t} \tilde{g}_{1}(x, \tau) d \tau\right\}\right)^{2}\left\|\tilde{\Pi}_{\vartheta_{\jmath}}\left(\begin{array}{c}
-P_{0} \\
\mathscr{M} P
\end{array}\right) J w\right\|^{2} \\
& \leq\left(\exp \left\{-\nu \int_{t_{0}}^{t} \tilde{g}_{1}(x, \tau) d \tau\right\}\right)^{2}\left\|\left(\begin{array}{c}
-P_{0} \\
\mathscr{L} P
\end{array}\right) J w\right\|^{2},
\end{aligned}
$$

which proves (4.17) and completes the proof of Lemma 4.3.

\section{The $L^{\infty}$ A-priori Estimates}

In this section we first prove an $L^{\infty}$-estimate which is basic for the results of this paper. At the end of this section we use this estimate to prove Theorem 2.5 and Corollary 2.6. Before we can state the estimate, we introduce some notations used throughout this section.

For $W=\left(w^{\left(-k_{0}\right)}, \ldots, w^{\left(k_{0}\right)}, s, z\right) \in \mathscr{Z}(\mathbb{R} \times[0, T)),(x, t) \in \mathbb{R} \times[0, T), 0 \leq \tau<T$ and $-k_{0} \leq k \leq k_{0}$ we set

$$
W^{(k)}(x, t)= \begin{cases}w^{(k)}(x, t), & k \neq 0 \\ \left(w^{(0)}(x, t), s(x, t)\right), & k=0\end{cases}
$$

and

$$
G_{k}(\tau ; x, t)=g_{1}\left(|P \sigma-P s|, z_{1}\right)\left(x+\lambda^{(k)}(\tau-t), \tau\right) .
$$

Moreover, for this $W$ let $W^{\prime}=\left(W^{\left(-k_{0}\right)}, \ldots, W^{\left(k_{0}\right)}\right)$,

$$
\left\|W^{\prime}(x, t)\right\|^{2}=\sum_{k=-k_{0}}^{k_{0}}\left\|W^{(k)}(x, t)\right\|^{2}
$$

and

$$
\left\|W^{\prime}\right\|_{\infty, t}=\sup _{\substack{x \in \mathbb{R} \\ 0 \leq \tau<t}}\left\|W^{\prime}(x, \tau)\right\| .
$$

Here $\left\|W^{(k)}(x, t)\right\|$ is the norm of $\mathbb{R}^{3} \times \mathscr{S}^{3}$ when $k \neq 0$ and of $\mathbb{R}^{3} \times \mathscr{S}^{3} \times \mathscr{S}^{3}$ when $k=0$. From the definitions given before Lemma 2.3 it is clear that

$$
\left\|W^{\prime}(x, t)\right\|=\left\|\left(w^{\left(-k_{0}\right)}, \ldots, w^{\left(k_{0}\right)}, s\right)(x, t)\right\| .
$$

The same conventions are also used for $W \in \mathscr{H}(\mathbb{R})$, where (5.3) is replaced by

$$
\left\|W^{\prime}\right\|_{\infty}=\sup _{x \in \mathbb{R}}\left\|W^{\prime}(x)\right\| .
$$

The a-priori estimate is

Lemma 5.1. Let the functions $g_{1}$ and $g_{2}$ satisfy the hypotheses of Theorem 1.1. Then there exists a constant $\mathscr{P}$ with the following property: Let $T>0$ and let 
$W=\left(w^{\left(-k_{0}\right)}, \ldots, w^{\left(k_{0}\right)}, s, z\right) \in \mathscr{Y}(\mathbb{R} \times[0, T))$ be a weak solution of (2.37)-(2.41) to the initial data $W^{0}=\left(w^{0\left(-k_{0}\right)}, \ldots, w^{0\left(k_{0}\right)}, s^{0}, z^{0}\right) \in \mathscr{Y}(\mathbb{R})$. Then we have for all $(x, t) \in \mathbb{R} \times[0, T)$ and all $|k| \leq k_{0}$,

$$
\left\|W^{(k)}(x, t)\right\| \leq\left[1+\mathscr{P} \max _{0 \leq \tau \leq t} G_{k}(\tau ; x, t)^{\omega} K^{*}(t, E(0, W))\right]\left\|\left(W^{0}\right)^{\prime}\right\|_{\infty}
$$

with $K^{*}(t, E(0, W))$ defined as in Theorem 1.1 and with

$$
\omega= \begin{cases}\frac{2}{2+\varrho}, & \text { when } g_{2} \equiv 0 \\ 1, & \text { otherwise } .\end{cases}
$$

The a-priori estimate in Theorem 2.5 will be a corollary of this estimate.

We now prove Lemma 5.1. The proof is in several steps and needs several lemmas. At first we need a representation formula for the solution $W$.

Representation Formula. In this section we always assume that

$$
W=\left(w^{\left(-k_{0}\right)}, \ldots, z\right) \in \mathscr{H}(\mathbb{R} \times[0, T))
$$

is a weak solution of (2.37)-(2.41) to the initial data $W^{0}=\left(w^{0\left(-k_{0}\right)}, \ldots, z^{0}\right) \in \mathscr{Y}(\mathbb{R})$. From (4.1) and (4.3) it follows for $(x, t) \in \mathbb{R} \times[0, T)$ and $k \neq 0$ that

$$
\begin{aligned}
w^{(k)}\left(x+\lambda^{(k)}(\tau-t), \tau\right)= & -\int_{0}^{\tau} \hat{F}_{k}(\eta, \tau ; x, t) a\left(x+\lambda^{(k)}(\eta-t), \eta\right) d \eta \\
& +\hat{F}_{k}(0, \tau ; x, t) w^{0(k)}\left(x-\lambda^{(k)} t\right) .
\end{aligned}
$$

To prove this formula it suffices to differentiate this equation with respect to $\tau$. Using (4.4) and (4.5) and noting that $w^{0(k)}(x) \in Y_{k}$ and $a(x, t) \in Y_{k}$, which is a consequence of the definition of $P_{k}$ in (2.36), it follows that (4.1) is satisfied and that $w^{(k)}\left(x-\lambda^{(k)} t, 0\right)=w^{0(k)}\left(x-\lambda^{(k)} t\right)$. This justifies (5.5), since solutions of (4.1) with given initial data are unique. Setting $\tau=t$ in (5.5) we obtain with (4.9) and (5.2) for $k \neq 0$ that

$$
\begin{aligned}
w^{(k)}(x, t)= & W^{(k)}(x, t) \\
= & -\int_{0}^{t} F_{k}(\tau ; x, t) G_{k}(\tau ; x, t) P_{k}\left[J \sum_{\substack{j=-k_{0} \\
j \neq k}}^{k_{0}} w^{(\jmath)}-s\right]\left(x+\lambda^{(k)}(\tau-t), \tau\right) d \tau \\
& +F_{k}(0 ; x, t) w^{0(k)}\left(x-\lambda^{(k)} t\right) .
\end{aligned}
$$

An analogous formula for $W^{(0)}=\left(w^{(0)}, s\right)$ is obtained from (4.2), (4.3) and (4.6)(4.9). To write this formula and (5.6) in a unified way, let for integers $k, j$ with $-k_{0} \leq k, j \leq k_{0}$ the operator $P_{k \jmath}$ be defined by

$$
P_{k j}= \begin{cases}P_{k} J & k, j \neq 0 \\ P_{k}(J,-I) & k \neq 0, j=0 \\ \left(P_{0}\right) . I . & k=0 .\end{cases}
$$


with $\left(\begin{array}{c}P_{0} \\ -\mathscr{M} P\end{array}\right)$ defined as in (4.17) and with

$$
(w, s) \mapsto(J,-I)(w, s)=(J,-I)\left(\begin{array}{c}
w \\
s
\end{array}\right)=J w-s: \mathbb{R}^{3} \times \mathscr{S}^{3} \times \mathscr{S}^{3} \rightarrow \mathscr{S}^{3} .
$$

With (5.1) we then obtain for $-k_{0} \leq k \leq k_{0}$ and $(x, t) \in \mathbb{R} \times[0, T)$ that

$$
\begin{aligned}
W^{(k)}(x, t)= & -\int_{0}^{t} F_{k}(\tau ; x, t) G_{k}(\tau ; x, t) \sum_{\substack{\jmath=-k_{0} \\
j \neq k}}^{k_{0}} P_{k j} W^{(\jmath)}\left(x+\lambda^{(k)}(\tau-t), \tau\right) d \tau \\
& +F_{k}(0 ; x, t) W^{0(k)}\left(x-\lambda^{(k)} t\right) .
\end{aligned}
$$

We can apply this formula recursively. To simplify the notation in the resulting formula, let for $j, k \in\left\{-k_{0}, \ldots, k_{0}\right\}$ with $j \neq k$,

$$
H_{j k}(\tau ; x, t)=-F_{j}(\tau ; x, t) G_{j}(\tau ; x, t) P_{\jmath k} .
$$

For a non-negative integer $n$ and for $-k_{0} \leq k \leq k_{0}$ let

$$
\begin{aligned}
M_{n}(k)=\{ & \left\{j=\left(j_{0}, \ldots, j_{n+1}\right) \in\left\{-k_{0}, \ldots, k_{0}\right\}^{n+2}: j_{0}=k\right. \\
& \text { and } \left.j_{l} \neq j_{l+1} \text { for all } l=0,1, \ldots, n\right\} .
\end{aligned}
$$

For $j=\left(j_{0}, \ldots, j_{n+1}\right) \in M_{n}(k), x \in \mathbb{R}$ and $0 \leq t_{n+1} \leq t_{n} \leq \ldots \leq t_{0}<T$ define

$$
\begin{array}{r}
L_{n}\left(x, t_{0}, t_{1}, \ldots, t_{n+1}, j\right)=H_{j_{0} \jmath_{1}}\left(t_{1} ; x, t_{0}\right) H_{\jmath_{1} j_{2}}\left(t_{2} ; x+\lambda^{\left(\jmath_{0}\right)}\left(t_{1}-t_{0}\right), t_{1}\right) \ldots \\
\ldots H_{\jmath_{n} \jmath_{n+1}}\left(t_{n+1} ; x+\sum_{l=0}^{n-1} \lambda^{\left(j_{l}\right)}\left(t_{l+1}-t_{l}\right), t_{n}\right) .
\end{array}
$$

Recursive application of (5.8) yields for every non-negative integer $n,-k_{0} \leq k \leq k_{0}$ and $\left(x, t_{0}\right) \in \mathbb{R} \times[0, T)$ the representation formula

$$
\begin{aligned}
W^{(k)}\left(x, t_{0}\right)= & \sum_{j \in M_{n}(k)} \int_{0}^{t_{0}} \ldots \int_{0}^{t_{n}} L_{n}\left(x, t_{0}, \ldots, t_{n+1}, j\right) \\
& \times W^{\left(\jmath_{n+1}\right)}\left(x+\sum_{l=0}^{n} \lambda^{\left(j_{l}\right)}\left(t_{l+1}-t_{l}\right), t_{n+1}\right) d t_{n+1} \ldots d t_{1} \\
& +\sum_{m=0}^{n-1} \sum_{\jmath \in M_{m}(k)} \int_{0}^{t_{0}} \ldots \int_{0}^{t_{m}} L_{m}\left(x, t_{0}, \ldots, t_{m+1}, j\right) \\
& \times F_{j_{m+1}}\left(0 ; x+\sum_{l=0}^{m} \lambda^{\left(\jmath_{l}\right)}\left(t_{l+1}-t_{l}\right), t_{m+1}\right) \\
& \times W^{0\left(\jmath_{m+1}\right)}\left(x+\sum_{l=0}^{m} \lambda^{\left(j_{l}\right)}\left(t_{l+1}-t_{l}\right)-\lambda^{\left(\jmath_{m+1}\right)} t_{m+1}\right) d t_{m+1} \ldots d t_{1} \\
& +F_{k}\left(0 ; x, t_{0}\right) W^{0(k)}\left(x-\lambda^{(k)} t_{0}\right) .
\end{aligned}
$$


Lemma 5.1 is proved by estimating the different terms in this formula. The necessary estimates are derived in a sequence of lemmas.

Lemma 5.2. Let $g_{1}: \mathbb{R}_{0}^{+} \times \mathbb{R} \rightarrow \mathbb{R}_{0}^{+}$be continuous and assume that there are constants $M_{1}^{*}, M_{2}^{*}>0,0<\varrho<2$ with

$$
g_{1}(\eta, \zeta) \leq M_{1}^{*} \eta^{\varrho}+M_{2}^{*}
$$

for all $\eta \geq 0, \zeta \in \mathbb{R}$. Let

$$
\begin{aligned}
r & =\frac{1}{2}+\frac{1}{\varrho} \\
\mathscr{P} & =\max \left\{\left\|P_{j l}\right\|:-k_{0} \leq j, l \leq k_{0}, j \neq l\right\}, \\
\Lambda & =2 \operatorname{P}^{2 r} \frac{\left(2 M_{1}^{*}\right)^{2 r-1}+\left(2 M_{2}^{*}\right)^{2 r}}{\min _{j \neq k}\left|\lambda^{(j)}-\lambda^{(k)}\right|}\left(L^{-1} \max _{|l| \leq k_{0}}\left|\lambda^{(l)}\right|+1\right) .
\end{aligned}
$$

For $j, k, l \in\left\{-k_{0}, \ldots, k_{0}\right\}$ with $j \neq k, j \neq l$ and for $0 \leq t_{0}<T$ we then have

$$
\int_{0}^{t_{0}} \int_{0}^{t_{1}}\left\|H_{j l}\left(t_{2} ; x+\lambda^{(k)}\left(t_{1}-t_{0}\right), t_{1}\right)\right\|^{2 r} d t_{2} d t_{1} \leq \Lambda\left(t_{0}+1\right)^{2}(E(0, W)+L) .
$$

The norm in the integrand in (5.17) is the operator norm of $H_{\jmath l}$.

Proof. From the definition of $H_{\jmath l}$ in (5.9) and from (4.14), (4.16), (5.15) we obtain

$$
\left\|H_{\jmath l}(\tau ; y, t)\right\| \leq G_{j}(\tau ; y, t)\left\|P_{j k}\right\| \leq \mathscr{P} G_{\jmath}(\tau ; y, t) .
$$

Therefore

$$
\begin{aligned}
& \int_{0}^{t_{0}} \int_{0}^{t_{1}}\left\|H_{\jmath l}\left(t_{2} ; x+\lambda^{(k)}\left(t_{1}-t_{0}\right), t_{1}\right)\right\|^{2 r} d t_{2} d t_{1} \\
& \leq \mathscr{P}^{2 r} \int_{0}^{t_{0}} \int_{0}^{t_{1}} G_{j}\left(t_{2} ; x+\lambda^{(k)}\left(t_{1}-t_{0}\right), t_{1}\right)^{2 r} d t_{2} d t_{1} \\
& =\mathscr{P}^{2 r} \int_{0}^{t_{0}} \int_{0}^{t_{1}}\left\{\left[g\left(|P \sigma-P s|, z_{1}\right)\right]\left(x+\lambda^{(k)}\left(t_{1}-t_{0}\right)+\lambda^{(j)}\left(t_{2}-t_{1}\right), t_{2}\right)\right\}^{2 r} d t_{2} d t_{1} \\
& =\mathscr{P}^{2 r}{ }_{N}
\end{aligned}
$$

For $0 \leq t_{2} \leq t_{1} \leq t_{0}$ let

$$
\kappa\left(t_{1}, t_{2}\right)=\left(x+\lambda^{(k)}\left(t_{1}-t_{0}\right)+\lambda^{(\jmath)}\left(t_{2}-t_{1}\right), t_{2}\right),
$$

and let $\Delta\left(x, t_{0}\right)$ be the triangle with vertex $\left(x, t_{0}\right)$ bounded by the lines $t_{1} \mapsto$ $x+\lambda^{(k)}\left(t_{1}-t_{0}\right), t_{2} \mapsto x+\lambda^{(j)}\left(t_{2}-t_{0}\right)$, and by the line $t=0$. Then

$$
\kappa:\left\{\left(t_{1}, t_{2}\right): 0 \leq t_{2} \leq t_{1} \leq t_{0}\right\} \rightarrow \Delta\left(x, t_{0}\right)
$$

and

$$
\operatorname{det}\left(\frac{\partial \kappa\left(t_{1}, t_{2}\right)}{\partial\left(t_{1}, t_{2}\right)}\right)=\lambda^{(k)}-\lambda^{(j)} \neq 0
$$


since by assumption $j \neq k$, whence

$$
N=\left|\lambda^{(k)}-\lambda^{(\jmath)}\right|^{-1} \int_{\Delta\left(x, t_{0}\right)}\left\{\left[g_{1}\left(|P \sigma-P s|, z_{1}\right)\right](\xi, \tau)\right\}^{2 r} d(\xi, \tau) .
$$

Let

$$
\Delta_{1}=\left\{(\xi, \tau) \in \Delta\left(x, t_{0}\right):\left[g_{1}\left(|P \sigma-P s|, z_{1}\right)\right](\xi, \tau) \geq 2 M_{2}^{*}\right\} .
$$

From (5.13) we then obtain for $(\xi, \tau) \in \Delta_{1}$,

$$
2 M_{2}^{*} \leq g_{1}\left(|P \sigma-P s|, z_{1}\right)(\xi, \tau) \leq M_{1}^{*}|P \sigma-P s|^{\varrho}+M_{2}^{*},
$$

hence

$$
g_{1}\left(|P \sigma-P s|, z_{1}\right)(\xi, \tau) \leq 2 M_{1}^{*}|P \sigma-P s|^{\varrho}
$$

and therefore

$$
\left[g_{1}\left(|P \sigma-P s|, z_{1}\right)(\xi, \tau)\right]^{2 r} \leq g_{1}\left(|P \sigma-P s|, z_{1}\right)(\xi, \tau)\left(2 M_{1}^{*}|P \sigma-P s|^{\varrho}\right)^{2 r-1}
$$

Because of $\varrho(2 r-1)=2$ we obtain from this estimate and from (5.20) that

$$
\begin{aligned}
N \leq & \left|\lambda^{(k)}-\lambda^{(j)}\right|^{-1}\left[\left(2 M_{1}^{*}\right)^{2 r-1} \int_{\Delta_{1}} g_{1}\left(|P \sigma-P s|, z_{1}\right)\right. \\
& \left.\times|P \sigma-P s|^{2} d(\xi, \tau)+\int_{\Delta\left(x, t_{0}\right) \backslash \Delta_{1}}\left(2 M_{2}^{*}\right)^{2 r} d(\xi, \tau)\right] \\
\leq & \frac{\left(2 M_{1}^{*}\right)^{2 r-1}+\left(2 M_{2}^{*}\right)^{2 r}}{\left|\lambda^{(k)}-\lambda^{(j)}\right|}\left[\int_{\Delta\left(x, t_{0}\right)} g_{1}\left(|P \sigma-P s|, z_{1}\right)\right. \\
& \left.\times|P \sigma-P s|^{2} d(\xi, \tau)+\int_{\Delta\left(x, t_{0}\right)} d(\xi, \tau)\right] .
\end{aligned}
$$

Since $W \in \mathscr{Y}(\mathbb{R} \times[0, T))$, it follows from Lemma 2.3 that $(\sigma, s, z)$ satisfies (2.4), which implies

$$
\left[g_{1}\left(|P \sigma-P s|, z_{1}\right)|P \sigma-P s|^{2}\right](x, t)=\left[g_{1}\left(|P \sigma-P s|, z_{1}\right)|P \sigma-P s|^{2}\right](-x, t),
$$

and since $(\sigma, s, z)$ is periodic with period $2 L$, we obtain from Lemma 3.2 that

$$
\int_{0}^{t} \int_{y-L}^{y+L} g_{1}\left(|P \sigma-P s|, z_{1}\right)|P \sigma-P s|^{2} d(\xi, \tau) \leq 2 E(0, W)
$$

for every $(y, t) \in \mathbb{R} \times[0, T)$. Let $n$ be the smallest integer with

$$
t_{0} \max _{-k_{0} \leq l \leq k_{0}}\left|\lambda^{l}\right| \leq n L .
$$

Then the triangle $\Delta\left(x, t_{0}\right)$ is contained in the union of $n$ disjoint strips of the form $[y-L, y+L] \times[0, \infty)$. From (5.21) and (5.22) we therefore obtain

$$
N \leq \frac{\left(2 M_{1}^{*}\right)^{2 r-1}+\left(2 M_{2}^{*}\right)^{2 r}}{\left|\lambda^{(k)}-\lambda^{(j)}\right|}\left[2 n E(0, W)+2 n L t_{0}\right]
$$


The estimate (5.17) is a consequence of this estimate, of (5.19) and of

$$
n \leq\left(L^{-1} \max _{|l| \leq k_{0}}\left|\lambda^{(l)}\right|+1\right)\left(t_{0}+1\right)
$$

which results from (5.23).

Lemma 5.3. Let the hypotheses of Lemma 5.2 be satisfied, let $q>0$, and let $j, k, l$ be integers with $-k_{0} \leq j, k, l \leq k_{0}, k \neq j, k \neq l$. Then

$$
\begin{aligned}
& \left.\int_{0}^{t_{0}} \| H_{\jmath k}\left(t_{1} ; x, t_{0}\right)\right) \|\left(t_{1}+1\right)^{q}\left(\int_{0}^{t_{1}}\left\|H_{k l}\left(t_{2} ; x+\lambda^{(j)}\left(t_{1}-t_{0}\right), t_{1}\right)\right\|^{2 r} d t_{2}\right)^{\frac{1}{2 r}} d t_{1} \\
& \leq(\Lambda(E(0)+L))^{\frac{1}{2 r}} \frac{1}{\left(q \frac{r}{r-1}+1\right)^{1-1 / r}}\left(t_{0}+1\right)^{q+1} \\
& \quad \times\left(\int_{0}^{t_{0}}\left\|H_{\jmath k}\left(t_{1} ; x, t_{0}\right)\right\|^{2 r} d t_{1}\right)^{\frac{1}{2 r}} .
\end{aligned}
$$

Proof. To simplify the notation we set

$$
\Psi_{\jmath k}(\tau ; y, t)=\left\|H_{j k}(\tau ; y, t)\right\|
$$

Let $r=\frac{1}{2}+\frac{1}{\varrho}>1$ be the constant from (5.14) and let $r^{\prime}$ satisfy

$$
\frac{1}{2 r}+\frac{1}{2 r^{\prime}}=1
$$

whence

$$
2 r^{\prime}=\frac{2 r}{2 r-1}<2 r
$$

Also, let $p, p^{\prime}$ satisfy

$$
\frac{1}{p}+\frac{1}{p^{\prime}}=1, \quad 2 r^{\prime} p=2 r
$$

hence

$$
p=\frac{2 r}{2 r^{\prime}}=2 r-1>1, \quad p^{\prime}=\frac{2 r-1}{2 r-2}>1 .
$$


We apply Hölder's inequality twice and use Lemma 5.2 to obtain

$$
\begin{aligned}
& \int_{0}^{t_{0}} \Psi_{j k}\left(t_{1} ; x, t_{0}\right)\left(t_{1}+1\right)^{q}\left(\int_{0}^{t_{1}} \Psi_{k l}\left(t_{2} ; x+\lambda^{(j)}\left(t_{1}-t_{0}\right), t_{1}\right)^{2 r} d t_{2}\right)^{\frac{1}{2 r}} d t_{1} \\
& \leq\left\{\int_{0}^{t_{0}}\left[\Psi_{\jmath k}\left(t_{1} ; x, t_{0}\right)\left(t_{1}+1\right)^{q}\right]^{2 r^{\prime}} d t_{1}\right\}^{\frac{1}{2 r^{\prime}}} \\
& \times\left\{\int_{0}^{t_{0}} \int_{0}^{t_{1}} \Psi_{k l}\left(t_{2} ; x+\lambda^{(j)}\left(t_{1}-t_{0}\right), t_{1}\right)^{2 r} d t_{2} d t_{1}\right\}^{\frac{1}{2 r}} \\
& \leq\left\{\int_{0}^{t_{0}}\left[\Psi_{\jmath k}\left(t_{1} ; x, t_{0}\right)\left(t_{1}+1\right)^{q}\right]^{2 r^{\prime}} d t_{1}\right\}^{\frac{1}{2 r^{\prime}}}\left[\Lambda\left(t_{0}+1\right)^{2}(E(0)+L)\right]^{\frac{1}{2 r}} \\
& \leq\left(\int_{0}^{t_{0}} \Psi_{\jmath k}\left(t_{1} ; x, t_{0}\right)^{2 r^{\prime} p} d t_{1}\right)^{\frac{1}{2 r^{\prime} p}}\left[\int_{0}^{t_{0}}\left(t_{1}+1\right)^{q 2 r^{\prime} p^{\prime}} d t_{1}\right]^{\frac{1}{2 r^{\prime} p^{\prime}}} \\
& \times\left[\Lambda\left(t_{0}+1\right)^{2}(E(0)+L)\right]^{\frac{1}{2 r}} \\
& \leq\left[\Lambda\left(t_{0}+1\right)^{2}(E(0)+L)\right]^{\frac{1}{2 r}}\left(\int_{0}^{t_{0}} \Psi_{j k}\left(t_{1} ; x, t_{0}\right)^{2 r} d t_{1}\right)^{\frac{1}{2 r}} \\
& \times\left(\int_{0}^{t_{0}}\left(t_{1}+1\right)^{q \frac{r}{r-1}} d t_{1}\right)^{\frac{r-1}{r}} \\
& \leq\left[\Lambda\left(t_{0}+1\right)^{2}(E(0)+L)\right]^{\frac{1}{2 r}} \frac{1}{\left(q \frac{r}{r-1}+1\right)^{1-1 / r}\left(t_{0}+1\right)^{q+\frac{r-1}{r}}} \\
& \times\left(\int_{0}^{t_{0}} \Psi_{j k}\left(t_{1} ; x, t_{0}\right)^{2 r} d t_{1}\right)^{\frac{1}{2 r}}
\end{aligned}
$$

Lemma 5.4. Let the hypotheses of Lemma 5.2 be satisfied, and let $j, k, l$ be integers with $-k_{0} \leq j, k, l \leq k_{0}, k \neq j, k \neq l$. Then

$$
\begin{aligned}
& \int_{0}^{t_{0}} \int_{0}^{t_{1}}\left\|H_{j k}\left(t_{1} ; x, t_{0}\right) H_{k l}\left(t_{2} ; x+\lambda^{(j)}\left(t_{1}-t_{0}\right), t_{1}\right)\right\| d t_{2} d t_{1} \leq[\Lambda(E(0)+L)]^{\frac{1}{2 r}} \\
& \quad \times \frac{1}{\left(\frac{2 r-1}{2 r-2}+1\right)^{1-1 / r}}\left(t_{0}+1\right)^{2-\frac{1}{2 r}}\left(\int_{0}^{t_{0}}\left\|H_{j k}\left(t_{1} ; x, t_{0}\right)\right\|^{2 r} d t_{1}\right)^{\frac{1}{2 r}} .
\end{aligned}
$$


Proof. Let $r^{\prime}$ be the constant from (5.25) and let $\Psi_{\jmath k}$ be the function from (5.24). Hölder's inequality yields

$$
\begin{aligned}
& \int_{0}^{t_{0}} \int_{0}^{t_{1}}\left\|H_{j k}\left(t_{1} ; x, t_{0}\right) H_{k l}\left(t_{2} ; x+\lambda^{(j)}\left(t_{1}-t_{0}\right), t_{1}\right)\right\| d t_{2} d t_{1} \\
& \leq \int_{0}^{t_{0}} \int_{0}^{t_{1}} \Psi_{j k}\left(t_{1} ; x, t_{0}\right) \Psi_{k l}\left(t_{2} ; x+\lambda^{(j)}\left(t_{1}-t_{0}\right), t_{1}\right) d t_{2} d t_{1} \\
& \leq \int_{0}^{t_{0}} \Psi_{j k}\left(t_{1} ; x, t_{0}\right)\left[\int_{0}^{t_{1}} \Psi_{k l}\left(t_{2} ; x+\lambda^{(j)}\left(t_{1}-t_{0}\right), t_{1}\right)^{2 r} d t_{2}\right]^{\frac{1}{2 r}}\left[\int_{0}^{t_{1}} d t_{2}\right]^{\frac{1}{2 r^{\prime}}} d t_{1} \\
& \leq \int_{0}^{t_{0}} \Psi_{j k}\left(t_{1} ; x, t_{0}\right)\left(t_{1}+1\right)^{\frac{1}{2 r^{\prime}}}\left(\int_{0}^{t_{1}} \Psi_{k l}\left(t_{2} ; x+\lambda^{(j)}\left(t_{1}-t_{0}\right), t_{1}\right)^{2 r} d t_{2}\right)^{\frac{1}{2 r}} d t_{1} .
\end{aligned}
$$

We use Lemma 5.3 with $q=\frac{1}{2 r^{\prime}}$ to estimate the right-hand side of this inequality and obtain the statement of the lemma.

Lemma 5.5. (i) Let $\mathscr{P}$ be defined as in (5.15) and let $j, k$ be integers with $-k_{0} \leq j$, $k \leq k_{0}, j \neq k$. Then

$$
\left(\int_{0}^{t_{0}}\left\|H_{j k}\left(t_{1} ; x, t_{0}\right)\right\|^{2 r} d t_{1}\right)^{\frac{1}{2 r}} \leq \mathscr{P} t_{0}^{\frac{1}{2 r}} \max _{0 \leq t_{1} \leq t_{0}} G_{j}\left(t_{1} ; x, t_{0}\right)
$$

(ii) If in addition $g_{2}(\eta, \zeta)=0$ for all $(\eta, \zeta) \in \mathbb{R}_{0}^{+} \times \mathbb{R}$, then

$$
\left(\int_{0}^{t_{0}}\left\|H_{j k}\left(t_{1} ; x, t_{0}\right)\right\|^{2 r} d t_{1}\right)^{\frac{1}{2 r}} \leq\left(2 r \mu_{0}\right)^{-\frac{1}{2 r}} \mathscr{P} \max _{0 \leq t_{1} \leq t_{0}} G_{\jmath}\left(t_{1} ; x, t_{0}\right)^{1-\frac{1}{2 r}}
$$

where $\mu_{0}=\min (\mu, \nu)$ with the constants $\mu$ from (4.15) and $\nu$ from (4.17).

Proof. (i) From (5.18) we obtain

$$
\begin{aligned}
\left(\int_{0}^{t_{0}}\left\|H_{\jmath k}\left(t_{1} ; x, t_{0}\right)\right\|^{2 r} d t_{1}\right)^{\frac{1}{2 r}} & \leq \mathscr{P}\left(\int_{0}^{t_{0}} G_{\jmath}\left(t_{1} ; x, t_{0}\right)^{2 r} d t_{1}\right)^{\frac{1}{2 r}} \\
& \leq \mathscr{P} t_{0}^{\frac{1}{2 r}} \max _{0 \leq t_{1} \leq t_{0}} G_{\jmath}\left(t_{1} ; x, t_{0}\right) .
\end{aligned}
$$


(ii) The definitions of $H_{j k}$ in (5.9), of $P_{j k}$ in (5.7), of $G_{j}$ in (5.2) and the estimates (4.15), (4.17) yield

$$
\begin{aligned}
& \int_{0}^{t_{0}}\left\|H_{j k}\left(t_{1} ; x, t_{0}\right)\right\|^{2 r} d t_{1} \\
& \leq \int_{0}^{t_{0}}\left[\exp \left\{-\mu_{0} \int_{t_{1}}^{t_{0}} G_{j}\left(\tau ; x, t_{0}\right) d \tau\right\}\left\|P_{\jmath k}\right\| G_{\jmath}\left(t_{1} ; x, t_{0}\right)\right]^{2 r} d t_{1} \\
& \leq \mathscr{P}^{2 r}\left[\max _{0 \leq t_{1} \leq t_{0}} G_{j}\left(t_{1} ; x, t_{0}\right)^{2 r-1}\right] \\
& \times \int_{0}^{t_{0}} \exp \left\{-2 r \mu_{0} \int_{t_{1}}^{t_{0}} G_{j}\left(\tau ; x, t_{0}\right) d \tau\right\} G_{j}\left(t_{1} ; x, t_{0}\right) d t_{1} \\
& =\mathscr{P}^{2 r}\left[\max _{0 \leq t_{1} \leq t_{0}} G_{j}\left(t_{1} ; x, t_{0}\right)^{2 r-1}\right] \frac{1}{2 r \mu_{0}} \\
& \times \int_{0}^{t_{0}}\left(-\frac{d}{d t_{1}} \exp \left\{-2 r \mu_{0} \int_{t_{1}}^{t_{0}} G_{j}\left(\tau ; x, t_{0}\right) d \tau\right\}\right) d t_{1} \\
& =\frac{\mathscr{P}^{2 r}}{2 r \mu_{0}}\left[\max _{0 \leq t_{1} \leq t_{0}} G_{\jmath}\left(t_{1} ; x, t_{0}\right)^{2 r-1}\right] \\
& \times\left(1-\exp \left\{-2 r \mu_{0} \int_{0}^{t_{0}} G_{j}\left(\tau ; x, t_{0}\right) d \tau\right\}\right),
\end{aligned}
$$

which implies statement (ii).

Proof of Lemma 5.1. We use Lemma 5.3-5.5 to estimate the terms in the representation formula (5.12). The definition of $L_{n}$ in (5.11) yields

$$
\begin{aligned}
\int_{0}^{t_{0}} \ldots & \int_{0}^{t_{n}}\left\|L_{n}\left(x, t_{0}, \ldots, t_{n+1}, j\right)\right\| d t_{n+1} \ldots d t_{1} \\
\leq & \int_{0}^{t_{0}}\left\|H_{\jmath_{0} \jmath_{1}}\left(t_{1} ; x, t_{0}\right)\right\| \int_{0}^{t_{1}}\left\|H_{j_{1} j_{2}}\left(t_{2} ; x+\lambda^{\left(j_{0}\right)}\left(t_{1}-t_{0}\right), t_{1}\right)\right\| \\
& \times \int_{0}^{t_{2}} \ldots \int_{0}^{t_{n-1}} \int_{0}^{t_{n}} \| H_{j_{n-1} \jmath_{n}}\left(t_{n} ; x+\sum_{l=0}^{n-2} \lambda^{\left(\jmath_{l}\right)}\left(t_{l+1}-t_{l}\right), t_{n-1}\right) \\
& \times H_{j_{n} j_{n+1}}\left(t_{n+1} ; x+\sum_{l=0}^{n-1} \lambda^{\left(\jmath_{l}\right)}\left(t_{l+1}-t_{l}\right), t_{n}\right) \| d t_{n+1} \ldots d t_{1} .
\end{aligned}
$$


The last term in this inequality can be estimated using Lemma 5.4. With

$$
y=x+\sum_{l=0}^{n-2} \lambda^{\left(\jmath_{l}\right)}\left(t_{l+1}-t_{l}\right)
$$

we obtain

$$
\begin{aligned}
& \int_{0}^{t_{n-1}} \int_{0}^{t_{n}}\left\|H_{j_{n-1} j_{n}}\left(t_{n} ; y, t_{n-1}\right) H_{j_{n} j_{n+1}}\left(t_{n+1} ; y+\lambda^{\left(j_{n-1}\right)}\left(t_{n}-t_{n-1}\right), t_{n}\right)\right\| d t_{n+1} d t_{n} \\
& \leq\left(\int_{0}^{t_{n-1}}\left\|H_{j_{n-1} j_{n}}\left(t_{n} ; y, t_{n-1}\right)\right\|^{2 r} d t_{n}\right)^{\frac{1}{2 r}} \\
& \quad \times \frac{\left(t_{n-1}+1\right)^{2-\frac{1}{2 r}}}{\left(1+\frac{2 r-1}{2 r-2}\right)^{1-1 / r}}[\Lambda(E(0)+L)]^{\frac{1}{2 r}}
\end{aligned}
$$

Invoking Lemma 5.3 the term resulting after insertion (5.27) into (5.26) can be estimated as follows:

$$
\begin{aligned}
\int_{0}^{t_{n-2}} \| & H_{J_{n-2} j_{n-1}}\left(t_{n-1} ; x+\sum_{l=0}^{n-3} \lambda^{\left(j_{l}\right)}\left(t_{l+1}-t_{l}\right), t_{n-2}\right) \| \\
& \times\left(\int_{0}^{t_{n-1}}\left\|H_{j_{n-1} j_{n}}\left(t_{n} ; x+\sum_{l=0}^{n-2} \lambda^{\left(j_{l}\right)}\left(t_{l+1}-t_{l}\right), t_{n-1}\right)\right\|^{2 r} d t_{n}\right)^{\frac{1}{2 r}} \\
& \times \frac{\left(t_{n-1}+1\right)^{2-\frac{1}{2 r}}}{\left(1+\frac{2 r-1}{2 r-2}\right)^{1-1 / r}} d t_{n-1}[\Lambda(E(0)+L)]^{\frac{1}{2 r}} \\
\leq & \left(\int_{0}^{t_{n-2} \| H_{j_{n-2} j_{n-1}}\left(t_{n-1} ; x+\sum_{l=0}^{n-3} \lambda^{\left(j_{l}\right)}\left(t_{l+1}-t_{l}\right), t_{n-2}\right)} \|^{2 r} d t_{n-1}\right)^{\frac{1}{2 r}} \\
& \times \frac{\left(t_{n-2}+1\right)^{3-\frac{1}{2 r}}[\Lambda(E(0)+L)]^{\frac{2}{2 r}}}{\left[\left(1+\left(1-\frac{1}{2 r}\right) \frac{r}{r-1}\right)\left(1+\left(2-\frac{1}{2 r}\right) \frac{r}{r-1}\right)\right]^{1-1 / r}},
\end{aligned}
$$

since

$$
1+\left(1-\frac{1}{2 r}\right) \frac{r}{r-1}=1+\frac{2 r-1}{2 r-2}
$$


It is clear that we now can apply Lemma 5.3 repeatedly to (5.26) and finally obtain for $n \geq 1$,

$$
\begin{aligned}
\int_{0}^{t_{0}} \ldots & \int_{0}^{t_{n}}\left\|L_{n}\left(x, t_{0}, \ldots, t_{n+1}, j\right)\right\| d t_{n+1} \ldots d t_{1} \\
\leq & \left(\int_{0}^{t_{0}}\left\|H_{j_{0} j_{1}}\left(t_{1} ; x, t_{0}\right)\right\|^{2 r} d t_{1}\right)^{\frac{1}{2 r}} \\
& \times \frac{\left(t_{0}+1\right)^{n+1-\frac{1}{2 r}}[\Lambda(E(0)+L)]^{\frac{n}{2 r}}}{\prod_{l=1}^{n}\left[1+\left(l-\frac{1}{2 r}\right) \frac{r}{r-1}\right]^{1-1 / r}}
\end{aligned}
$$

For $n=0$ we obtain from Hölder's inequality,

$$
\begin{aligned}
\int_{0}^{t_{0}}\left\|L_{0}\left(x, t_{0}, t_{1}, j\right)\right\| d t_{1} & =\int_{0}^{t_{0}}\left\|H_{\jmath_{0} \jmath_{1}}\left(t_{1} ; x, t_{0}\right)\right\| d t_{1} \\
& \leq\left(\int_{0}^{t_{0}}\left\|H_{j_{0} j_{1}}\left(t_{1} ; x, t_{0}\right)\right\|^{2 r} d t_{1}\right)^{\frac{1}{2 r}}\left(\int_{0}^{t_{0}} d t_{1}\right)^{\frac{1}{2 r^{\prime}}} \\
& \leq\left(\int_{0}^{t_{0}}\left\|H_{\jmath_{0} \jmath_{1}}\left(t_{1} ; x, t_{0}\right)\right\|^{2 r} d t_{1}\right)^{\frac{1}{2 r}}\left(t_{0}+1\right)^{1-\frac{1}{2 r}}
\end{aligned}
$$

which shows that (5.28) also holds for $n=0$.

Observe next that with the norm $\left\|W^{\prime}\right\|_{\infty, t_{0}}$ defined in (5.3), we obtain for $n \geq 0$,

$$
\begin{aligned}
& \| \int_{0}^{t_{0}} \ldots \int_{0}^{t_{n}} L_{n}\left(x, t_{0}, \ldots, t_{n+1}, j\right) W^{\left(\jmath_{n+1}\right)} \\
& \quad \times\left(x+\sum_{l=0}^{n} \lambda^{\left(j_{l}\right)}\left(t_{l+1}-t_{l}\right), t_{n+1}\right) d t_{n+1} \ldots d t_{1} \| \\
& \quad \leq \int_{0}^{t_{0}} \ldots \int_{0}^{t_{n}}\left\|L_{n}\left(x, t_{0}, \ldots, t_{n+1}, j\right)\right\| d t_{n+1} \ldots d t_{1}\left\|W^{\prime}\right\|_{\infty, t_{0}} .
\end{aligned}
$$

From (4.14) and (4.16) we conclude

$$
\left\|F_{j}(0 ; y, t) W^{0(j)}\left(y-\lambda^{(l)} t_{0}\right)\right\| \leq\left\|\left(W^{0}\right)^{\prime}\right\|_{\infty}
$$


which yields for $m \geq 0$,

$$
\begin{aligned}
& \| \int_{0}^{t_{0}} \ldots \int_{0}^{t_{m}} L_{m}\left(x, t_{0}, \ldots, t_{m+1}, j\right) F_{\jmath_{m+1}}\left(0 ; x+\sum_{l=0}^{m} \lambda^{\left(j_{l}\right)}\left(t_{l+1}-t_{l}\right), t_{m+1}\right) \\
& \quad \times W^{0\left(\jmath_{m+1}\right)}\left(x+\sum_{l=0}^{m} \lambda^{\left(\jmath_{l}\right)}\left(t_{l+1}-t_{l}\right)-\lambda^{\left(\jmath_{m}+1\right)} t_{m+1}\right) d t_{m+1} \ldots d t_{1} \| \\
& \leq \int_{0}^{t_{0}} \ldots \int_{0}^{t_{m}}\left\|L_{m}\left(x, t_{0}, \ldots, t_{m+1}, j\right)\right\| d t_{m+1} \ldots d t_{1}\left\|\left(W^{0}\right)^{\prime}\right\|_{\infty} .
\end{aligned}
$$

Taking into account that the set $M_{m}(k)$ defined in (5.10) contains $\left(2 k_{0}\right)^{m+1}$ elements, we infer from (5.12) and from (5.28)-(5.31) that

$$
\begin{aligned}
& \left\|W^{(k)}\left(x, t_{0}\right)\right\| \leq\left(\int_{0}^{t_{0}}\left\|H_{j_{0} \jmath_{1}}\left(t_{1} ; x, t_{0}\right)\right\|^{2 r} d t_{1}\right)^{\frac{1}{2 r}} \\
& \times\left[\frac{\left(2 k_{0}\right)^{n+1}\left(t_{0}+1\right)^{n+1-\frac{1}{2 r}}[\Lambda(E(0)+L)]^{\frac{n}{2 r}}}{\prod_{l=1}^{n}\left[1+\left(l-\frac{1}{2 r}\right) \frac{r}{r-1}\right]^{1-1 / r} \|_{\infty, t_{0}}}\right. \\
& \left.+\sum_{m=0}^{n-1} \frac{\left(2 k_{0}\right)^{m+1}\left(t_{0}+1\right)^{m+1-\frac{1}{2 r}}[\Lambda(E(0)+L)]^{\frac{m}{2 r}}}{\prod_{l=1}^{m}\left[1+\left(l-\frac{1}{2 r}\right) \frac{r}{r-1}\right]^{1-1 / r}}\left\|\left(W^{0}\right)^{\prime}\right\|_{\infty}\right] \\
& +\left\|\left(W^{0}\right)^{\prime}\right\|_{\infty} \cdot
\end{aligned}
$$

Because of

$$
1+\left(l-\frac{1}{2 r}\right) \frac{r}{r-1}=l+\frac{2 l-1}{2 r-2}+1 \geq l
$$

for $l \geq 1$, it follows

$$
\prod_{l=1}^{n}\left[1+\left(l-\frac{1}{2 r}\right) \frac{r}{r-1}\right]^{1-1 / r} \geq(n !)^{1-1 / r},
$$

which implies that the first term on the right-hand side of (5.32) tends to zero for $n \rightarrow \infty$. The estimate (5.4) of Lemma 5.1 is therefore obtained from (5.32) by letting $n \rightarrow \infty$ and using Lemma 5.5 to estimate the term

$$
\left(\int_{0}^{t_{0}}\left\|H_{\jmath_{0 J_{1}}}\left(t_{1} ; x, t_{0}\right)\right\|^{2 r} d t_{1}\right)^{\frac{1}{2 r}}
$$

remembering the definition of $r$ in (5.14) and remembering that $j_{0}=k$, by definition of $M_{m}(k)$. The proof of Lemma 5.1 is complete. 
Proof of Theorem 2.5. By Theorem 3.1 there exist to every initial data

$$
W^{0}=\left(w^{0\left(-k_{0}\right)}, \ldots, w^{0\left(k_{0}\right)}, s^{0}, z^{0}\right) \in \mathscr{Y}(\mathbb{R})
$$

satisfying (2.43) a $T>0$ and a weak solution

$$
W=\left(w^{\left(-k_{0}\right)}, \ldots, w^{\left(k_{0}\right)}, s, z\right) \in \mathscr{Y}(\mathbb{R} \times[0, T))
$$

of (2.37)-(2.41), which is locally unique. To show that (2.44) is satisfied, note first that the definition of $|\cdot|$ in the introduction and the definition of the scalar product $[\cdot, \cdot \cdot]$ in (2.1) imply for $k \neq 0$,

$$
\begin{aligned}
\left|J w^{(k)}\right|^{2} & =\left(J w^{(k)}, J w^{(k)}\right) \leq \delta\left(D^{-1} J w^{(k)}, J w^{(k)}\right) \\
& \leq \delta\left[w^{(k)}, w^{(k)}\right]=\delta\left\|w^{(k)}\right\|^{2}=\delta\left\|W^{(k)}\right\|^{2} \leq \delta\left\|W^{\prime}\right\|^{2}
\end{aligned}
$$

and

$$
\begin{aligned}
\left|J w^{(0)}\right|^{2}+|s|^{2} & \leq \delta\left(D^{-1} J w^{(0)}, J w^{(0)}\right)+\mathscr{C} \frac{1}{\mathscr{M} b}(s, s) \\
& \leq(\delta+\mathscr{M})\left(\left[w^{(0)}, w^{(0)}\right]+\frac{1}{\mathscr{M} b}(s, s)\right)=(\delta+\mathscr{M b})\left\|W^{(0)}\right\|^{2} \\
& \leq(\delta+\mathscr{M b})\left\|W^{\prime}\right\|^{2}
\end{aligned}
$$

where $\delta$ is the largest eigenvalue of the positive definite operator $D$. Since $P: \mathscr{S}^{3} \rightarrow$ $\mathscr{S}^{3}$ is an orthogonal projector with respect to the scalar product $(\cdot, \cdot \cdot)$, we thus obtain from these estimates and from (2.40) that

$$
\begin{aligned}
|P \sigma(x, t)-s(x, t)| & =\left|P J \sum_{k=-k_{0}}^{k_{0}} w^{(k)}-s\right| \leq \sum_{k=-k_{0}}^{k_{0}}\left|J w^{(k)}\right|+|s| \\
& \leq\left(2 k_{0}+2\right) \sqrt{\delta+\mathscr{C}}\left\|W^{\prime}(x, t)\right\| .
\end{aligned}
$$

Therefore (5.2), (5.3) and estimate (1.12) yield for $0 \leq \tau \leq t$ and for the constant $\omega$ from Lemma 5.1 that

$$
\begin{aligned}
\mathscr{P} G_{k}(\tau ; x, t)^{\omega} & \leq \mathscr{P}\left\{M_{1}^{*}\left[|P \sigma-P s|\left(x+\lambda^{(k)}(\tau-t), \tau\right)\right]^{\varrho}+M_{2}^{*}\right\}^{\omega} \\
& \leq \mathscr{P}\left\{M_{1}^{*}\left[\left(2 k_{0}+2\right) \sqrt{\delta+\mathscr{C}}\left\|W^{\prime}\left(x+\lambda^{(k)}(\tau-t), \tau\right)\right\|\right]^{\varrho}+M_{2}^{*}\right\}^{\omega} \\
& \leq \mathscr{P}\left\{M_{1}^{*}\left[\left(2 k_{0}+2\right) \sqrt{\delta+\mathscr{M} b}\left\|W^{\prime}\right\|_{\infty, t}\right]^{\varrho}+M_{2}^{*}\right\}^{\omega} \\
& \leq C_{1}\left\|W^{\prime}\right\|_{\infty, t}^{\varrho \omega}+C_{2}
\end{aligned}
$$

with $C_{1}=\mathscr{P}\left\{M_{1}^{*}\left[\left(2 k_{0}+2\right) \sqrt{\delta+\mathscr{C} b}\right]^{\varrho}\right\}^{\omega}, C_{2}=\mathscr{P} M_{2}^{*^{\omega}}$, wher we used that $\omega=1$ or $\omega=2(2+\varrho)^{-1}$, whence $\omega \leq 1$. Consequently, from the estimate of Lemma 5.1 we conclude for $0 \leq t<T$,

$$
\begin{aligned}
\left\|W^{\prime}\right\|_{\infty, t} & \leq \sup _{\substack{x \in \mathbb{R} \\
0 \leq \tau<t}} \sum_{k=-k_{0}}^{k_{0}}\left\|W^{(k)}(x, \tau)\right\| \\
& \leq\left(2 k_{0}+1\right)\left[1+\left(C_{1}\left\|W^{\prime}\right\|_{\infty, t}^{\varrho \omega}+C_{2}\right) K^{*}(t, E(0))\right]\left\|\left(W^{0}\right)^{\prime}\right\|_{\infty} \\
& \leq \frac{1}{2}\left[\tilde{M}_{3}\left(1+K^{*}\right)+\tilde{M}_{4} K^{*}\left\|W^{\prime}\right\|_{\infty, t}^{\varrho \omega}\right]\left\|\left(W^{0}\right)^{\prime}\right\|_{\infty},
\end{aligned}
$$


with $K^{*}=K^{*}(t, E(0))$ and

$$
\tilde{M}_{3}=2\left(2 k_{0}+1\right)\left(1+C_{2}\right), \quad \tilde{M}_{4}=2\left(2 k_{0}+1\right) C_{1} .
$$

Either we have $\tilde{M}_{4} K^{*}\left\|W^{\prime}\right\|_{\infty, t}^{\varrho \omega} \leq M_{3}^{*}\left(1+K^{*}\right)$, in which case (5.35) yields

$$
\left\|W^{\prime}\right\|_{\infty, t}=\tilde{M}_{3}\left(1+K^{*}\right)\left\|\left(W^{0}\right)^{\prime}\right\|_{\infty} .
$$

Otherwise we have

$$
\left\|W^{\prime}\right\|_{\infty, t} \leq \tilde{M}_{4} K^{*}\left\|W^{\prime}\right\|_{\infty, t}^{\varrho \omega}\left\|\left(W^{0}\right)^{\prime}\right\|_{\infty}
$$

whence

$$
\left\|W^{\prime}\right\|_{\infty, t} \leq\left(\tilde{M}_{4} K^{*}\left\|\left(W^{0}\right)^{\prime}\right\|_{\infty}\right)^{\frac{1}{1-\varrho \omega}}
$$

because the hypotheses of Theorem 1.1 and the definition of $\omega$ imply $0<\varrho<1$ and $\omega=1$, or $0<\varrho<2$ and $\omega=2(2+\varrho)^{-1}$, and therefore in both cases $\varrho \omega<1$. The estimates (5.36) and (5.37) together imply (2.44).

Proof of Corollary 2.6. Let $W \in \mathscr{H}(\mathbb{R} \times[0, T))$ be the local solution to the initial data $W^{0} \in \mathscr{Y} /(\mathbb{R})$ which exists according to Theorem 2.5 . We abbreviate the term on the right-hand side of (2.44) by $\Gamma\left(t, W^{0}\right)$. The differential equation (1.8) or, equivalently, (2.39), and the estimates (1.19), (2.44) and (5.33), (5.34) then imply

$$
\begin{aligned}
\partial_{t}|z|^{2} & =2 z \cdot \partial_{t} z=2 z \cdot h(z,|P \sigma-s|,|s|) \\
& \leq 2|z| c^{*}(|P \sigma-s|+|s|)(|s|+1) \leq 2 c^{*}\left(C_{3}\left\|W^{\prime}\right\|\right)\left(|z|^{2}+|z|\right) \\
& \leq 2 c^{*}\left(C_{3} \Gamma\left(t, W^{0}\right)\right) 2\left(|z|^{2}+1\right)
\end{aligned}
$$

since $|z| \leq|z|^{2}+1$, where $C_{3}=\left(2 k_{0}+3\right) \sqrt{\delta+M}$. Hence,

$$
\partial_{t} \ln \left(|z|^{2}+1\right) \leq 4 c^{*}\left(C_{3} \Gamma\left(t, W^{0}\right)\right)
$$

Integration yields

$$
|z(x, t)|^{2}+1 \leq\left(\left|z^{0}(x)\right|^{2}+1\right) e^{4 \int_{0}^{t} c^{*}\left(C_{3} \Gamma\left(\tau, W^{0}\right)\right) d \tau}
$$

which implies that

$$
z \in L^{\infty}(\mathbb{R} \times[0, T))
$$

whenever the solution $W$ exists on $\mathbb{R} \times[0, T)$. Now if the solution $W$ would not exist on $\mathbb{R} \times[0, \infty)$, then there would exist a maximal time of existence $T_{\infty}<\infty$, and therefore (3.1) would hold. But (3.1) contradicts (2.44) and (5.38), and consequently the solution exists on the domain $\mathbb{R} \times[0, \infty)$.

This completes the proofs of Theorem 2.5 and Corollary 2.6 and therefore also the proofs of Theorem 1.1 and Corollary 1.2. 


\section{Appendix}

We state the equations describing the viscoelastic deformation of metals in the form which derives directly from the model assumptions often used in engineering. We use the notations of Sect. 1 .

Let $B \subseteq \mathbb{R}^{3}$ be a bounded domain with smooth boundary $\partial B$, and let $u: B \times \mathbb{R}_{0}^{+} \rightarrow$ $\mathbb{R}^{3}$ be the displacement field. Then for $(x, t) \in B \times \mathbb{R}_{0}^{+}$,

$$
\begin{aligned}
\hat{\varrho} u_{t t}(x, t) & =\operatorname{div} \sigma(x, t) \\
e(x, t) & =\frac{1}{2}\left[\nabla_{x} u(x, t)+\left(\nabla_{x} u(x, t)\right)^{T}\right], \\
\sigma(x, t) & =D\left(e(x, t)-e^{n}(x, t)\right) .
\end{aligned}
$$

The boundary condition is

$$
\sigma(x, t) n(x)=0, \quad(x, t) \in \partial B \times \mathbb{R}_{0}^{+},
$$

where $n(x)$ denotes the exterior unit normal vector at $x \in \partial B, e(x, t)$ is the strain tensor, $e^{n}(x, t)$ is the tensor of inelastic strain. (A1) is a constitutive equation, but others are necessary to determine $e^{n}(x, t)$. To formulate such equations, the material is modelled as a system of springs and dashpots. This system is completely characterized by the equations

$$
\begin{aligned}
S(x, t) & =\sigma(x, t)-\frac{1}{3}(\operatorname{tr} \sigma(x, t)) I \\
S= & =s+s^{f} \\
e^{n} & =e^{a}+e^{p} \\
s & =\mathscr{M} b e^{a} \\
\frac{\partial}{\partial t} e^{n}(x, t) & =g_{1}\left(\left|s^{f}(x, t)\right|, z_{1}(x, t)\right) s^{f}(x, t), \\
\frac{\partial}{\partial t} e^{p}(x, t) & =g_{2}\left(|s(x, t)|, z_{2}(x, t)\right) s(x, t), \\
-\frac{\partial}{\partial t} z(x, t) & =-h\left(z(x, t),\left|s^{f}(x, t)\right|,|s(x, t)|\right) .
\end{aligned}
$$

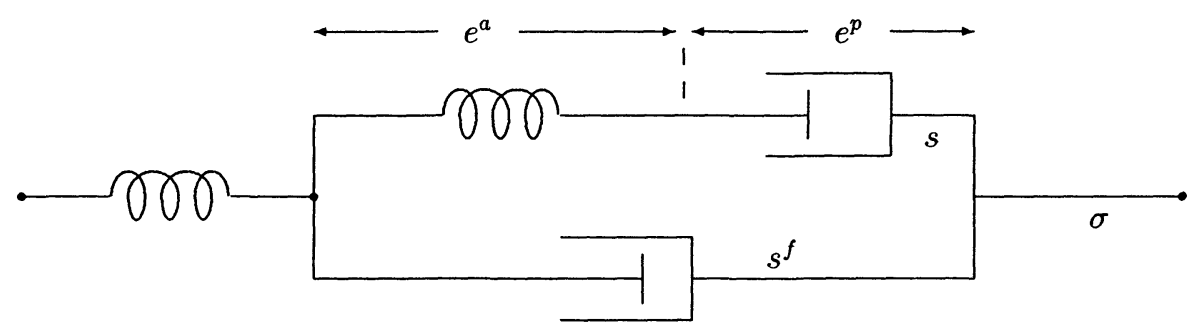

Fig. 1. 
Here $z=\left(z_{1}, z_{2}\right)$ are hardening parameters, $I$ is the identity matrix, $e^{a}, e^{p}$ are strain tensors, and $s, s^{f}$ are stress tensors. The equation (A2) is the constitutive equation of the spring, (A3) and (A3) are the constitutive equations of the two dashpots in the figure, and Eq. (A5) controls the evolution of the hardening parameters. The necessary initial conditions are

$$
\begin{gathered}
u(x, 0)=u^{0}(x), \quad u_{t}(x, 0)=v^{0}(x), \quad \sigma(x, 0)=\sigma^{0}(x), \\
s(x, 0)=s^{0}(x), \quad z(x, 0)=z^{0}(x) .
\end{gathered}
$$

Equations (1.1)-(1.5) follow from the equations stated here by combination.

\section{References}

1. Alber, H.-D.: On a system of equations from the theory of non-linear visco-plasticity. Preprint 1265, Fachbereich Mathematik der Technischen Hochschule Darmstadt 1989

2. Blanchard, D.: Nonlinear viscoelasticity with time-dependent coefficients. Nonlinear Anal., Theory Methods Appl. 10, 1391-1410 (1986)

3. Blanchard, D., Le Tallec, P., Ravachol, M.: Numerical analysis of evolution problems in nonlinear small strain elastoviscoplasticity. Numer. Math. 55, 177-195 (1989)

4. Duvaut, G., Lions, J.L.: Les inequations en mécanique et en physique, Paris: Dunod, 1972

5. Hart, E.W.: Constitutive relations for the nonelastic deformation of metals. Trans. ASME, J. Engng. Mat. Technol. 98, 193-202 (1976)

6. Johnson, C.: Existence theorems in plasticity. J. Math. Pures et Appl. 55, 431-444 (1976)

7. Johnson, C.: On plasticity with hardening. J. Math. Anal. Appl. 62, 325-336 (1978)

8. Le Tallec, P.: Numerical analysis of viscoelastic problems. Paris: Masson; Berlin, Heidelberg, New York: Springer, 1990

9. Lemaitre, J., Chaboche, J.L.: Mécanique des matériaux solides. Paris: Dunod, 1985

10. Miller, A.K.: An inelastic constitutive model for monotonic, cyclic and creep deformation: Part 1 - Equations development and analytical procedures. Trans. ASME, J. Eng. Mat. Technol. 98, 97-105 (1976)

11. Moreau, J.J.: On unilateral constraints, friction and plasticity. Bressanone: C.I.M.E. 1973

12. Moreau, J.J.: Application of convex analysis to the treatment of elasto-plastic systems. In: Springer Lecture Notes in Math. 503. P. Germain, B. Nayroles (eds.) Berlin, Heidelberg, New York: Springer 1975

13. Suquet, P.-M.: Evolution problems for a class of dissipative materials. Quart. Applied Math. 38, 391-414 (1982) 
